\title{
Genome-wide survey of potato MADS-box genes reveals that StMADS1 and StMADS13 are putative downstream targets of tuberigen StSP6A
}

Huhu Gao ${ }^{1}$, Ziming Wang ${ }^{2}$, Silu Li ${ }^{1}$, Menglu Hou' ${ }^{1}$ Yao Zhou' ${ }^{1}$, Yaqi Zhao ${ }^{1}$, Guojun Li ${ }^{1}$, Hua Zhao ${ }^{1 *}$ and Haoli Ma ${ }^{1 *}$ (D)

\begin{abstract}
Background: MADS-box genes encode transcription factors that are known to be involved in several aspects of plant growth and development, especially in floral organ specification. To date, the comprehensive analysis of potato MADSbox gene family is still lacking after the completion of potato genome sequencing. A genome-wide characterization, classification, and expression analysis of MADS-box transcription factor gene family was performed in this study.

Results: A total of 153 MADS-box genes were identified and categorized into MIKC subfamily (MIKC ${ }^{C}$ and MIKC $)$ and M-type subfamily ( $M a, M B$, and $M \gamma$ ) based on their phylogenetic relationships to the Arabidopsis and rice MADS-box genes. The potato M-type subfamily had 114 members, which is almost three times of the MIKC members (39), indicating that M-type MADS-box genes have a higher duplication rate and/or a lower loss rate during potato genome evolution. Potato MADS-box genes were present on all 12 potato chromosomes with substantial clustering that mainly contributed by the M-type members. Chromosomal localization of potato MADS-box genes revealed that MADS-box genes, mostly MIKC, were located on the duplicated segments of the potato genome whereas tandem duplications mainly contributed to the M-type gene expansion. The potato MIKC subfamily could be further classified into 11 subgroups and the T16-like, AGL17-like, and FLC-like subgroups found in Arabidopsis were absent in potato. Moreover, the expressions of potato MADS-box genes in various tissues were analyzed by using RNA-seq data and verified by quantitative real-time PCR, revealing that the MIKC ${ }^{C}$ genes were mainly expressed in flower organs and several of them were highly expressed in stolon and tubers. StMADS1 and StMADS13 were up-regulated in the StSP6A-overexpression plants and down-regulated in the StSP6A-RNAi plant, and their expression in leaves and/or young tubers were associated with high level expression of StSP6A.

Conclusion: Our study identifies the family members of potato MADS-box genes and investigate the evolution history and functional divergence of MADS-box gene family. Moreover, we analyze the MIKC ${ }^{C}$ expression patterns and screen for genes involved in tuberization. Finally, the StMADS1 and StMADS13 are most likely to be downstream target of StSP6A and involved in tuber development.
\end{abstract}

Keywords: Potato, MADS-box, Tuberigen, StSP6A, Tuberization

\footnotetext{
* Correspondence: zhaohua362@nwsuaf.edu.cn; mahaoli@nwsuaf.edu.cn ${ }^{1}$ College of Agronomy, Northwest A\&F University, Yangling 712100, Shaanxi, China

Full list of author information is available at the end of the article
}

(c) The Author(s). 2018 Open Access This article is distributed under the terms of the Creative Commons Attribution 4.0 International License (http://creativecommons.org/licenses/by/4.0/), which permits unrestricted use, distribution, and reproduction in any medium, provided you give appropriate credit to the original author(s) and the source, provide a link to the Creative Commons license, and indicate if changes were made. The Creative Commons Public Domain Dedication waiver (http://creativecommons.org/publicdomain/zero/1.0/) applies to the data made available in this article, unless otherwise stated. 


\section{Background}

The MADS-box gene family has been extensively studied for its important roles in transcriptional regulation in eukaryotes [1-3]. The word of MADS-box is an acronym for Mini chromosome maintenance 1 (MCM1) in yeast (Sacchromyces cerevisiae), AGAMOUS (AG) in Arabidopsis (Arabidopsis thaliana), DEFICIENS (DEF) in snapdragon (Antirrhinum majus) and serum response factor (SRF) in human (Homo sapiens) [4-7]. MADS-box genes are characterized by N-terminal conservative MADS-box domains that are approximately 58-60 amino acids in length, which functions in combination to DNA [7, 8]. In plants, MADS-box transcription factors are involved in almost every important process during plant growth and development $[9,10]$.

Based on phylogenetic relationship, MADS-box gene family has been divided into two major lineages in plants, type I and type II, which were resulting from an ancestral gene duplication [11, 12]. Type I genes are also named as M-type MADS-box genes, which contain three subgroups $(\mathrm{M} \alpha, \mathrm{M} \beta$, and $\mathrm{M} \gamma)$. The classical structure of M-type MADS-box genes is an N-terminal MADS domain and a relatively less conservative domain in the C-terminal [13]. In most plants, higher frequency of segmental gene duplications and weaker purifying selection result in a faster step of birth-and-death to type I genes compared to type II genes [14]. Type II MADS-box genes are also known as MIKC-type genes, which encode MEF2-like proteins [15]. In addition to the MADS domain, type II MADS-box genes contain three other domains, including intervening (I), kertain-like (K), and C-terminal (C) domains from $\mathrm{N}$-terminal to $\mathrm{C}$-terminal. The intervening (I) domain consists of approximately 30 amino acids and contributes to the dimerization of MADS-box proteins [16]. The kertain-like (K) domain is about 70 amino acids and more conservative than intervening (I) domain. The coiled-coil structure is significant to regulate the dimerization of MADS-box proteins. C-terminal (C) domain is a highly variable region in MADS-box proteins related to transcriptional activation and formation of protein complexes [17]. Type II MADS-box genes can be further classified into $\mathrm{MIKC}^{\mathrm{C}}$ (the ' $\mathrm{C}$ ' stands for 'Classic') and MIKC" based on the variable intervening (I) domain [18]. The domain compositions of these two subfamilies in type II are quite different. MIKC" subfamily exhibit a longer intervening (I) domain and less conservative kertain-like (K) domain [19]. Therefore, in early studies, MIKC" subfamily was attributed into M-type MADS-box genes named $M \delta[12]$.

The first MADS-box gene in plants was found to be related to the differentiation of flower [3]. ABCDE model had been successfully adopted to explain the determination of floral organ identity. Recent studies have also found that MIKC $^{\mathrm{C}}$ subfamily is related to photoperiod-regulated floral meristem identity, gametophyte development, sporophyte (diploid) generation, seed pigmentation, and embryo development [20-24]. Most of genes in ABCDE model belong to MIKC ${ }^{\mathrm{C}}$ subfamily $[6,25,26]$. Besides, MADS-box genes in $\mathrm{MIKC}^{\mathrm{C}}$ subfamily plays irreplaceable biological functions in the stress-responsive processes, for instance, TaMADS2 was up-regulated in response to wheat stripe rust infection [27].

The functions of MIKC* MADS-box genes are less elucidated than those in $\mathrm{MIKC}^{\mathrm{C}}$ subfamily and it is found that the heterodimers of MIKC*-type proteins are essential for the pollen maturation and pollen tube growth in Arabidopsis [28]. In potato, only three MADS-box genes have been previously reported, they are potato MADS-box 1-1 (POTM1-1), StMADS11, and StMADS16 [29, 30]. POTM1-1 gene expression is temporally and spatially regulated in both vegetative and floral organs, transcriptional suppression of POTM1-1 activates axillary meristem development by increasing the cytokinin levels [29, 31, 32]. StMADS11 is expressed in all vegetative tissues of the potato plant, mainly in the stem, but not in flower organs [33]. Ectopic expression of StMADS16 modifies the inflorescence structure by increasing both internode length and flower proliferation of the inflorescence meristems and confers vegetative features to the flower [29]. Recent study finds that FLOWERING LOCUS T in potato (StSP6A) is a mobile signal for potato tuberization. StSP6A, homologs of FT in Arabidopsis, is very likely to control tuberization through regulating the expressions of downstream MADS-box genes [34, 35]. Therefore, there is an urgent need to characterize the MADS-box gene family in potato and screen for MADS-box candidates involved in tuberization. The complete genome sequencing of the potato in 2011 enabled us to perform a genome-wide identification of MADS-box genes in potato [36].

In this study, multiple bioinformatics methods were applied to perform a comprehensive survey of MADS-box genes in potato. In addition, the gene structure, phylogenetic relationships, chromosomal locations, conserved motifs and tissue-specific expressions of MADS-box genes were investigated in potato. Our work would be useful in helping to establish the basic information of MADS-box genes in potato and in screening out several MADS-box genes related to tuberization and following tuber development.

\section{Methods}

\section{Identification of MADS-box genes in potato}

The potato genome sequence data used for the identification and annotation of StMADS genes was downloaded from Potato Genome Sequencing Consortium (PGSC, http://potato.plantbiology.msu.edu/). BLASTP, InerPro ID and keyword searches were performed to obtain the putative MADS-box genes in potato. First of all, the known Arabidopsis MADS-box protein sequences were used as 
query to perform BLASTP utility against the potato protein database (PGSC_DM_v3.4_pep_nonredundant.fasta) in local computer with an expected value cutoff of $1 \mathrm{e}-3$. Then, InterPro ID (IPR003340) and keyword searches (MADS-box) were also applied to identify putative potato MADS-box proteins in PGSC database by online searching. All putative MADS-box sequences were collected and the redundant sequences were manually removed, the remaining candidate MADS-box sequences were submitted to NCBI Conserved Domain (CD) search (https:// www.ncbi.nlm.nih.gov/Structure/cdd/wrpsb.cgi) to confirm the existence of MADS-box domain.The gene structure of MADS genes was drawn with TB tools (http://cj-chen. github.io/tbtools/) using GFF3 files downloaded from PGSC.

\section{Chromosomal location and gene duplication}

MapChart 2.2 was exploited to draw the gene location in the physical map of potato MADS-box genes [37]. Potato MADS-box genes were named based on the position information obtained from PGSC with concerning about already reported POTM1-1 (StMADS1), StMADS11, and StMADS16. According to the nomenclature method used in rice, the remaining potato MADS-box genes were named as StMADS2 to StMADS153 followed the order of

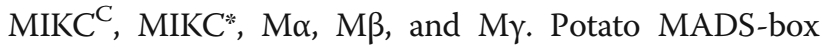
genes without chromosomal positions were named at the last of the list. Tandem duplicated genes were determined in PGSC with a criterion that no more than five genes between two genes with high homology (>50\%). Segmental duplicated genes of potato were obtained from the Plant Genome Duplication Database (PGDD, http://chibba.pgml.uga.edu/duplication/).

\section{Phylogenetic and conserved motif analyses of potato MADS-box genes}

Potato, rice, and Arabidopsis MADS-box protein sequences were aligned using ClustalX version 1.83. The phylogenetic trees were generated by using Neighbor joining (NJ) method in MEGA6.06 with bootstrap value 1000 replicates to evaluate the significance of the nodes. To ensure that the divergent domains could contribute to the topology of the NJ tree, pairwise gap deletion mode was used to construct the tree. Moreover, the potato MADS-box protein sequences were submitted to MEME (http://meme-suite.org/) to determine the conserved motif in these sequences.

\section{Phylogenetic analysis of MADS-box genes between potato and tomato}

To investigate the phylogenetic relationships of MADS-box genes between potato and tomato, a genome-wide search against the Solanum lycopersicum proteome (Solanum lycopersicum Annotation Release 103, ftp://ftp.ncbi.nlm.nih.gov/
genomes/Solanum_lycopersicum/protein/) using blastp program in dos environment of windows. The threshold of e-value was set for $1 \mathrm{e}-3$. The candidate gene was submitted to InterPro to exclude genes without MADS-box domain. A phylogenetic tree was generated to determine the relationship of MADS genes in these two species using the protein sequence aligned with Muscle program in MEGA7.0 and the same method mentioned above.

\section{Expression analyses based on publicly available RNA-seq and microarray data}

The expression patterns of potato MADS-box gene family members were determined using the data deposited in the PGSC database which derived from Illumina RNA-seq of a wide range of developmental stages [36]. The expression profiles of genes on POCI (Potato Oligo Chip Initiative) microarrays were previously performed in stolons of StSP6A-overexpression and StSP6A-RNAi plants [34]. The MADS-box genes were used as queries to search DNA probes using BLASTN and the results of MADS-box genes were used for further study with e-value set for 1e-3. The probes were selected if they were annotated as MADS-box family members by the microarray platform and were assigned to corresponding potato MADS-box genes if their identity were $100 \%$ with exceptions of single nucleotide polymorphism (SNP). The deduced FPKM value of these genes acquired from published data were normalized with $\log 2$ to make it suitable for the further visualized in Pretty Heatmap (http://www.ehbio.com/ImageGP/index.php/Home/Index/PHeatmap.html).

\section{Plant materials collection and qRT-PCR}

The plants of potato cultivar 'Desire' was cultivated in greenhouse of Northwest A\&F University from March to June $\left(23 \pm 2{ }^{\circ} \mathrm{C}, 16 \mathrm{~h}\right.$ light $/ 8 \mathrm{~h}$ dark). The different tissues and organs were collected at different time after sprouting. Stem, leaf, and flower were sampled at flowering, whereas stolons and young tubers were collected ten days after flowering. In addition, mature tubers were taken 90 days after sprouting. All samples were immediately frozen in liquid nitrogen and stored at $-80{ }^{\circ} \mathrm{C}$ until used. Total RNA was extracted using a high purity total RNA rapid extraction kit (BioTeke, RP1202, China) and first-strand cDNA was synthesized using a ReverTra Ace Kit (TOYOBO, FSK-100, Japan) following the manufacturer's instructions.

Primer 5.0 was used to design gene-specific primers of MADS-box in potato (Additional file 1: Table S1). Real-time quantitative RT-PCR was performed by using the SYBR green mix (KAPA, KK4601, USA) in a Real-time PCR machine (BioRad, CFX96, USA). The internal reference gene was ef1 $\alpha$ and three biological replicates were used to estimate the expression level by the method of two stand curves as described previously [38]. 


\section{Results \\ Identification and comparative analysis of MADS genes in potato}

Three bioinformatics methods were used to identify the MADS-box genes in potato. A local BLASTP search was performed with a cutoff e-value of 1e-3 by using the Arabidopsis MADS-box proteins as query, which resulted in 169 MADS-box candidates. The keyword and InterPro ID (IPR003340) searches against the PGSC website resulted in 145 and 156 MADS-box candidates, respectively. These candidates were submitted to NCBI CDD to confirm the existence of MADS-box domain. After removing the redundant sequences, 153 total MADS-box genes were found in potato. The names of StMADS1, StMADS11, and StMADS16 were introduced by previous reports and remaining genes were named from StMADS2 to 153 (except for StMADS11 and StMADS16) according to their chromosomal locations and subfamily affiliation (Table 1). Based on phylogenetic relationships of Arabidopsis, rice, and potato MADS-box proteins (Fig. 1), 153 potato MADS-box proteins were classified into two subfamily MIKC and M-type. In potato, the number of MADS-box genes in MIKC subfamily was 39 and this subfamily was mainly comprised of two subgroups, 30 MADS-box genes in MIKC ${ }^{\mathrm{C}}$ and 9 MADS-box genes in MIKC*. The number of MADS-box genes in M-type is 114 and this subfamily contained three subgroups, 70 MADS-box genes in $M \alpha, 28$ MADS-box genes in $M \beta$, and 16 MADS-box genes in My.

MIKC subfamily members are about 200 amino acid in length and contain more exons than those of M-type subfamily (Table 1 ). The MIKC family members have an average of 6.4 exon number, and $86.7 \%$ of them contain more than 5. But for the M-type, most of them (106 of 114) have only one exon (Table 1). These results about exon number in MADS-box genes are similar to those have been reported in Arabidopsis, rice, cucumber, and apple. The exon-intron structures of MIKC members are more complex than those of M-type.

The total number of MADS-box genes in 10 species that had been previously reported was quite different (Table 2) [12, 39-49]. The reported numbers of MADS-box genes were from 43 to 167, which were positively correlated to corresponding genome size except for that of Arabidopsis (Table 2). Generally, MIKC subfamily consisted of more members than M-Type subfamily as reported in previous studies, but we found that the number of M-type MADS-box genes (114) is approximately three times to that of MIKC MADS-box genes (39) in potato (Table 2). In the other nine species, the number of MIKC subfamily members was close to or more than that of M-type subfamily members.

\section{Chromosomal distribution and duplication events of StMADS genes}

The MapChart software was used to map the physical position of MADS-box genes on 12 chromosomes of potato, which would be helpful for us to perform further study of function of MADS-box genes in potato (Fig. 2). Based on the information of chromosomal locations of potato MADS-box genes, it was found that seven genes were not localized to the chromosomes of potato, five of which belonged to the M-type subfamily (Table 1 ). The rest MADS-box genes (146) were distributed on the 12 chromosomes and the top five chromosomes with more MADS-box genes are Chr01 (31 genes), Chr04 (25 genes), Chr05 (15 genes), Chr11 (14 genes), and Chr03 (10) (Fig. 2).

To further explore the distribution patterns of MADS-box genes, a radar map was exhibited to show the distributions of each subfamily in 12 chromosomes. It was found that substantial clustering was detected in each of at least four chromosomes which was mainly contributed by the gene number of M-type subfamily rather than MIKC subfamily, implying there may be a selective expansion pattern mainly happened in the M-type subfamily (Fig. 3). The MADS-box genes belong to MIKC subfamily distributed on all chromosomes except on Chr09 (Fig. 2). For the M-type MADS-box genes, $52.9 \%$ MADS-box genes of the M $\alpha$ subgroup was clustered on Chr01 and Chr04.

Moreover, the gene duplication events in the MADS-box gene family were analyzed and it was found that $47.7 \%$ (73 of 153) MADS-box genes derived from gene duplications (Figs. 2 and 4a). Tandem duplicated genes were mainly located on chromosome 1 and chromosome 5, accounting for about $51.9 \%$ of tandem duplicated genes. $78.9 \%$ (45 of 57) tandem duplicated genes of belonged to the M-type, indicating that tandem duplications played an important role in the expansion of M-type family genes. 31.5\% (12 of 38) genes of the MIKC subfamily were resulted from tandem duplications. Interestingly, it was found that tandem duplications could occur between different subgroups (e.g. StMADS44-46 belonged to $M \alpha$ and StMADS111 belonged to $M \beta$ ), indicating that gene duplication not only contributed to the expansion of MADS-box gene family but also lead to functional diversifications.

Compared with tandem duplications, segmental duplications only accounted for $10.5 \%$ of the total MADS-box genes in potato (Fig. 4a). 26.7\% (8 of 30) of MIKC ${ }^{\mathrm{C}}$ MADS-box genes were resulted from segmental duplications. Those genes were located in $\mathrm{Chr02}$ (two genes), Chr03 (two genes), Chr04 (one genes), Chr05 (two genes), and Chr11 (one genes). We found, interestingly, three copies of the segmental duplicated gene pair (StMADS17 and 18, StMADS13 and 14, StMADS8 and 9), among which StMADS9, 14 and 17 are from SEP group, while StMADS8, 
Table 1 The detailed information of potato MADS-box gene family. Genes with an asterisk tail means identified in previous studies

\begin{tabular}{|c|c|c|c|c|c|}
\hline Name & Locus & Chromosomal locations & Protein (aa) & Exon & Subfamily \\
\hline StMADS1 $^{*}$ & PGSC0003DMG400004081 & chr06 $51191112-51198207$ & 250 & 8 & $M I K C^{C}$ \\
\hline StMADS2 & PGSC0003DMG401006771 & chr01 64818874-64826057 & 287 & 8 & MIKC $C^{C}$ \\
\hline StMADS3 & PGSC0003DMG400000008 & chr01 71388491-71392334 & 224 & 8 & $\mathrm{MIKC}^{\mathrm{C}}$ \\
\hline StMADS4 & PGSC0003DMG400000136 & chr01 $71421222-71426490$ & 255 & 8 & $\mathrm{MIKC}^{\mathrm{C}}$ \\
\hline StMADS5 & PGSC0003DMG400026563 & chr02 24953367-24959857 & 234 & 8 & MIKC ${ }^{C}$ \\
\hline StMADS6 & PGSC0003DMG400028442 & chr02 31027789-31034809 & 248 & 9 & $\mathrm{MIKC}^{\mathrm{C}}$ \\
\hline StMADS7 & PGSC0003DMG400003541 & chr02 39864994-39869063 & 225 & 7 & $\mathrm{MIKC}^{\mathrm{C}}$ \\
\hline StMADS8 & PGSC0003DMG400001378 & chr02 $45341112-45345702$ & 247 & 8 & $\mathrm{MIKC}^{\mathrm{C}}$ \\
\hline StMADS9 & PGSC0003DMG400001377 & chr02 45353007-45357495 & 246 & 8 & MIKC C \\
\hline StMADS10 & PGSC0003DMG401024252 & chr03 41578383-41583244 & 119 & 3 & $\mathrm{MIKC}^{\mathrm{C}}$ \\
\hline StMADS11 $1^{*}$ & PGSC0003DMG400033570 & chr01 82400439-82408299 & 221 & 9 & MIKC C \\
\hline StMADS12 & PGSC0003DMG401015205 & chr03 50457307-50460580 & 200 & 8 & MIKC $C^{C}$ \\
\hline StMADS13 & PGSC0003DMG400024625 & chr03 54879384-54886641 & 248 & 8 & $\mathrm{MIKC}^{\mathrm{C}}$ \\
\hline StMADS14 & PGSC0003DMG400024626 & chr03 54896515-54901975 & 246 & 8 & $\mathrm{MIKC}^{\mathrm{C}}$ \\
\hline StMADS15 & PGSC0003DMG400003709 & chr04 70409561-70413264 & 228 & 7 & $\mathrm{MIKC}^{\mathrm{C}}$ \\
\hline StMADS16 $6^{*}$ & PGSC0003DMG400009363 & chr04 64959661-64967188 & 235 & 11 & MIKC $C^{C}$ \\
\hline StMADS17 & PGSC0003DMG400028359 & chr05 757295-763364 & 242 & 8 & $\mathrm{MIKC}^{\mathrm{C}}$ \\
\hline StMADS18 & PGSC0003DMG400028358 & chr05 766418-772401 & 244 & 8 & $\mathrm{MIKC}^{\mathrm{C}}$ \\
\hline StMADS19 & PGSC0003DMG400021899 & chr05 14664987-14671873 & 241 & 8 & MIKC $C^{C}$ \\
\hline StMADS20 & PGSC0003DMG400025279 & chr06 $36656761-36660368$ & 111 & 3 & $\mathrm{MIKC}^{\mathrm{C}}$ \\
\hline StMADS21 & PGSC0003DMG400005176 & chr06 47282868-47291175 & 232 & 9 & $\mathrm{MIKC}^{\mathrm{C}}$ \\
\hline StMADS22 & PGSC0003DMG400017295 & chr07 51027282-51036001 & 254 & 6 & $\mathrm{MIKC}^{\mathrm{C}}$ \\
\hline StMADS23 & PGSC0003DMG401007392 & chr08 40704456-40708461 & 210 & 7 & MIKC $C^{C}$ \\
\hline StMADS24 & PGSC0003DMG400022748 & chr08 53768699-53776641 & 224 & 7 & MIKC $C^{C}$ \\
\hline StMADS25 & PGSC0003DMG400010263 & chr10 $38698247-38706939$ & 214 & 6 & $\mathrm{MIKC}^{\mathrm{C}}$ \\
\hline StMADS26 & PGSC0003DMG400023729 & chr10 57811655-57814557 & 229 & 7 & $\mathrm{MIKC}^{\mathrm{C}}$ \\
\hline StMADS27 & PGSC0003DMG400016203 & chr11 2317625-2323465 & 238 & 8 & $\mathrm{MIKC}^{\mathrm{C}}$ \\
\hline StMADS28 & PGSC0003DMG400025525 & chr11 16127866-16137240 & 232 & 7 & $\mathrm{MIKC}^{\mathrm{C}}$ \\
\hline StMADS29 & PGSC0003DMG401018787 & chr11 16991875-16995488 & 116 & 4 & $\mathrm{MIKC}^{\mathrm{C}}$ \\
\hline StMADS30 & PGSC0003DMG400001938 & chr00 12593321-12596548 & 188 & 2 & MIKC C \\
\hline StMADS31 & PGSC0003DMG400019525 & chr03 55865548-55867778 & 142 & 3 & $\mathrm{MIKC}^{*}$ \\
\hline StMADS32 & PGSC0003DMG400038617 & chr04 31598124-31598495 & 84 & 2 & MIKC ${ }^{*}$ \\
\hline StMADS33 & PGSC0003DMG400036414 & chr04 $52111872-52112228$ & 82 & 2 & $\mathrm{MIKC}^{*}$ \\
\hline StMADS34 & PGSC0003DMG400044568 & chr04 52327361-52331720 & 158 & 3 & MIKC ${ }^{*}$ \\
\hline StMADS35 & PGSC0003DMG400045842 & chr06 35336837-35338244 & 151 & 5 & $\mathrm{MIKC}^{*}$ \\
\hline StMADS36 & PGSC0003DMG401026197 & chr07 47364697-47367540 & 155 & 6 & MIKC ${ }^{*}$ \\
\hline StMADS37 & PGSC0003DMG403026197 & chr07 47437818-47445958 & 94 & 9 & $\mathrm{MIKC}^{*}$ \\
\hline StMADS38 & PGSC0003DMG400017759 & chr12 55492383-55493047 & 108 & 2 & MIKC $C^{*}$ \\
\hline StMADS39 & PGSC0003DMG400017760 & chr12 55554242-55562194 & 225 & 3 & $\mathrm{MIKC}^{*}$ \\
\hline StMADS40 & PGSC0003DMG400038539 & chr01 14769002-14769334 & 110 & 1 & Ma \\
\hline StMADS41 & PGSC0003DMG400045528 & chr01 40043124-40043513 & 129 & 1 & Ma \\
\hline StMADS42 & PGSC0003DMG400011328 & chr01 57376759-57377670 & 303 & 1 & $\mathrm{Ma}$ \\
\hline StMADS43 & PGSC0003DMG400011316 & chr01 57795443-57796789 & 421 & 1 & $\mathrm{Ma}$ \\
\hline StMADS44 & PGSC0003DMG400006290 & chr01 60247610-60248203 & 197 & 1 & Ma \\
\hline
\end{tabular}


Table 1 The detailed information of potato MADS-box gene family. Genes with an asterisk tail means identified in previous studies (Continued)

\begin{tabular}{|c|c|c|c|c|c|}
\hline Name & Locus & Chromosomal locations & Protein (aa) & Exon & Subfamily \\
\hline StMADS45 & PGSC0003DMG400041956 & chr01 60265275-60265868 & 197 & 1 & $\mathrm{Ma}$ \\
\hline StMADS46 & PGSC0003DMG400046706 & chr01 60293060-60293646 & 189 & 2 & $\mathrm{Ma}$ \\
\hline StMADS47 & PGSC0003DMG400000062 & chr01 72596658-72597790 & 143 & 1 & $\mathrm{Ma}$ \\
\hline StMADS48 & PGSC0003DMG400040999 & chr01 75519222-75519740 & 172 & 1 & $\mathrm{Ma}$ \\
\hline StMADS49 & PGSC0003DMG400022465 & chr01 75665917-75666411 & 495 & 1 & $\mathrm{Ma}$ \\
\hline StMADS50 & PGSC0003DMG400022464 & chr01 75669936-75670403 & 155 & 1 & $\mathrm{Ma}$ \\
\hline StMADS51 & PGSC0003DMG400037739 & chr01 75677724-75678191 & 155 & 1 & Ma \\
\hline StMADS52 & PGSC0003DMG400022462 & chr01 75682108-75682572 & 154 & 1 & $\mathrm{Ma}$ \\
\hline StMADS53 & PGSC0003DMG400022460 & chr01 75693397-75693888 & 163 & 1 & $\mathrm{Ma}$ \\
\hline StMADS54 & PGSC0003DMG400044662 & chr01 75699473-75699940 & 155 & 1 & $\mathrm{Ma}$ \\
\hline StMADS55 & PGSC0003DMG400042000 & chr01 75715667-75716134 & 155 & 1 & $\mathrm{Ma}$ \\
\hline StMADS56 & PGSC0003DMG400035140 & chr01 75719828-75720178 & 116 & 1 & $\mathrm{Ma}$ \\
\hline StMADS57 & PGSC0003DMG400005119 & chr01 83295937-83296952 & 213 & 1 & $\mathrm{Ma}$ \\
\hline StMADS58 & PGSC0003DMG400044387 & chr01 83297295-83297960 & 221 & 1 & $\mathrm{Ma}$ \\
\hline StMADS59 & PGSC0003DMG400005131 & chr01 83300154-83300747 & 197 & 1 & $\mathrm{Ma}$ \\
\hline StMADS60 & PGSC0003DMG400046652 & chr02 14861994-14862299 & 101 & 1 & $\mathrm{Ma}$ \\
\hline StMADS61 & PGSC0003DMG400038730 & chr02 15687958-15688452 & 164 & 1 & $\mathrm{Ma}$ \\
\hline StMADS62 & PGSC0003DMG400013012 & chr02 22892178-22892726 & 182 & 1 & $\mathrm{Ma}$ \\
\hline StMADS63 & PGSC0003DMG400039595 & chr02 25493501-25494016 & 171 & 1 & $\mathrm{Ma}$ \\
\hline StMADS64 & PGSC0003DMG400042442 & chr03 3618726-3619148 & 140 & 1 & $\mathrm{Ma}$ \\
\hline StMADS65 & PGSC0003DMG400037384 & chr03 3660635-3661183 & 182 & 1 & $\mathrm{Ma}$ \\
\hline StMADS66 & PGSC0003DMG400045551 & chr03 4972791-4973312 & 173 & 1 & $\mathrm{Ma}$ \\
\hline StMADS67 & PGSC0003DMG400046651 & chr03 13264973-13265449 & 158 & 1 & $\mathrm{Ma}$ \\
\hline StMADS68 & PGSC0003DMG400037338 & chr04 750699-751226 & 175 & 1 & $\mathrm{Ma}$ \\
\hline StMADS69 & PGSC0003DMG400019742 & chr04 9343623-9346031 & 90 & 6 & $\mathrm{Ma}$ \\
\hline StMADS70 & PGSC0003DMG400040226 & chr04 21299801-21300325 & 174 & 1 & $\mathrm{Ma}$ \\
\hline StMADS71 & PGSC0003DMG400041035 & chr04 27499096-27499626 & 176 & 1 & $\mathrm{Ma}$ \\
\hline StMADS72 & PGSC0003DMG400036160 & chr04 27559777-27560277 & 166 & 1 & $\mathrm{Ma}$ \\
\hline StMADS73 & PGSC0003DMG400044513 & chr04 27753241-27753636 & 131 & 1 & $\mathrm{Ma}$ \\
\hline StMADS74 & PGSC0003DMG400040625 & chr04 27761388-27761732 & 114 & 1 & $\mathrm{Ma}$ \\
\hline StMADS75 & PGSC0003DMG400035221 & chr04 27826362-27826868 & 168 & 1 & $\mathrm{Ma}$ \\
\hline StMADS76 & PGSC0003DMG400036006 & chr04 28232462-28232779 & 105 & 1 & $\mathrm{Ma}$ \\
\hline StMADS77 & PGSC0003DMG400034832 & chr04 28463661-28464235 & 164 & 2 & $\mathrm{Ma}$ \\
\hline StMADS78 & PGSC0003DMG400040127 & chr04 28566215-28566718 & 167 & 1 & $\mathrm{Ma}$ \\
\hline StMADS79 & PGSC0003DMG400045239 & chr04 29232278-29232646 & 122 & 1 & $\mathrm{Ma}$ \\
\hline StMADS80 & PGSC0003DMG400042688 & chr04 49390684-49391226 & 180 & 1 & $\mathrm{Ma}$ \\
\hline StMADS81 & PGSC0003DMG400044761 & chr04 49734932-49735474 & 180 & 1 & $\mathrm{Ma}$ \\
\hline StMADS82 & PGSC0003DMG400039153 & chr04 54460837-54461322 & 161 & 1 & $\mathrm{Ma}$ \\
\hline StMADS83 & PGSC0003DMG400037581 & chr04 54575543-54576028 & 161 & 1 & $\mathrm{Ma}$ \\
\hline StMADS84 & PGSC0003DMG400024799 & chr04 58551552-58552247 & 231 & 1 & $\mathrm{Ma}$ \\
\hline StMADS85 & PGSC0003DMG400040743 & chr06 10343031-10343519 & 162 & 1 & $\mathrm{Ma}$ \\
\hline StMADS86 & PGSC0003DMG400040848 & chr06 45773429-45773971 & 180 & 1 & $\mathrm{Ma}$ \\
\hline StMADS87 & PGSC0003DMG400043595 & chr07 15176598-15177149 & 183 & 1 & $\mathrm{Ma}$ \\
\hline
\end{tabular}


Table 1 The detailed information of potato MADS-box gene family. Genes with an asterisk tail means identified in previous studies (Continued)

\begin{tabular}{|c|c|c|c|c|c|}
\hline Name & Locus & Chromosomal locations & Protein (aa) & Exon & Subfamily \\
\hline StMADS88 & PGSC0003DMG400004482 & chr08 38320573-38321088 & 171 & 1 & $\mathrm{Ma}$ \\
\hline StMADS89 & PGSC0003DMG400029147 & chr08 38625243-38625695 & 150 & 1 & Ma \\
\hline StMADS90 & PGSC0003DMG400039537 & chr09 4843300-4843812 & 170 & 1 & $\mathrm{Ma}$ \\
\hline StMADS91 & PGSC0003DMG400036125 & chr09 6381512-6381946 & 144 & 1 & $\mathrm{Ma}$ \\
\hline StMADS92 & PGSC0003DMG400043218 & chr09 8426313-8426747 & 144 & 1 & $\mathrm{Ma}$ \\
\hline StMADS93 & PGSC0003DMG400038182 & chr09 8460217-8460714 & 165 & 1 & $\mathrm{Ma}$ \\
\hline StMADS94 & PGSC0003DMG400043614 & chr09 28872055-28872590 & 149 & 2 & $\mathrm{Ma}$ \\
\hline StMADS95 & PGSC0003DMG400040285 & chr09 45285005-45285310 & 101 & 1 & $\mathrm{Ma}$ \\
\hline StMADS96 & PGSC0003DMG400037372 & chr09 $45311443-45311877$ & 144 & 1 & $\mathrm{Ma}$ \\
\hline StMADS97 & PGSC0003DMG400019393 & chr10 6655038-6655631 & 173 & 1 & $\mathrm{Ma}$ \\
\hline StMADS98 & PGSC0003DMG400043633 & chr10 13335280-13335894 & 204 & 1 & $\mathrm{Ma}$ \\
\hline StMADS99 & PGSC0003DMG400035501 & chr10 16298995-16299315 & 106 & 1 & $\mathrm{Ma}$ \\
\hline StMADS100 & PGSC0003DMG400042890 & chr10 43539248-43539784 & 178 & 1 & $\mathrm{Ma}$ \\
\hline StMADS101 & PGSC0003DMG400039674 & chr10 $43571982-43572518$ & 178 & 1 & $\mathrm{Ma}$ \\
\hline StMADS102 & PGSC0003DMG400036190 & chr11 36057399-36057959 & 186 & 1 & $\mathrm{Ma}$ \\
\hline StMADS103 & PGSC0003DMG400047285 & chr11 36128116-36128568 & 150 & 1 & $\mathrm{Ma}$ \\
\hline StMADS104 & PGSC0003DMG400039835 & chr11 36737512-36737970 & 152 & 1 & $\mathrm{Ma}$ \\
\hline StMADS105 & PGSC0003DMG400043978 & chr11 41767865-41768500 & 211 & 1 & $\mathrm{Ma}$ \\
\hline StMADS106 & PGSC0003DMG400038207 & chr11 41778980-41779333 & 117 & 1 & $\mathrm{Ma}$ \\
\hline StMADS107 & PGSC0003DMG400001143 & chr11 41867026-41867556 & 176 & 1 & $\mathrm{Ma}$ \\
\hline StMADS108 & PGSC0003DMG400044457 & chr12 52437112-52437654 & 180 & 1 & $\mathrm{Ma}$ \\
\hline StMADS109 & PGSC0003DMG400046733 & chr00 38424953-38425450 & 165 & 1 & $\mathrm{Ma}$ \\
\hline StMADS110 & PGSC0003DMG400046736 & chr01 44165290-44165835 & 181 & 1 & $M \beta$ \\
\hline StMADS111 & PGSC0003DMG400037342 & chr01 60364849-60365394 & 181 & 1 & $M \beta$ \\
\hline StMADS112 & PGSC0003DMG400024916 & chr01 80241931-80242893 & 320 & 1 & $M \beta$ \\
\hline StMADS113 & PGSC0003DMG400024902 & chr01 80529617-80530232 & 139 & 1 & $M \beta$ \\
\hline StMADS114 & PGSC0003DMG400005968 & chr04 1911128-1911634 & 168 & 1 & $M \beta$ \\
\hline StMADS115 & PGSC0003DMG400035463 & chr05 9324497-9325042 & 181 & 1 & $M \beta$ \\
\hline StMADS116 & PGSC0003DMG400007682 & chr05 9990538-9991511 & 157 & 1 & $M \beta$ \\
\hline StMADS117 & PGSC0003DMG400018623 & chr05 10026168-10026958 & 219 & 1 & $M \beta$ \\
\hline StMADS118 & PGSC0003DMG400041411 & chr05 51418059-51418661 & 200 & 1 & $M \beta$ \\
\hline StMADS119 & PGSC0003DMG400047062 & chr06 8761350-8762057 & 235 & 1 & $M \beta$ \\
\hline StMADS120 & PGSC0003DMG400026582 & chr06 44289139-44289651 & 131 & 1 & $M \beta$ \\
\hline StMADS121 & PGSC0003DMG400033092 & chr06 52148889-52149384 & 131 & 1 & $M \beta$ \\
\hline StMADS122 & PGSC0003DMG400027886 & chr07 39107047-39108380 & 299 & 2 & $M \beta$ \\
\hline StMADS123 & PGSC0003DMG400027613 & chr11 12890859-12892025 & 388 & 1 & $M \beta$ \\
\hline StMADS124 & PGSC0003DMG400035247 & chr11 13686758-13687444 & 228 & 1 & $M \beta$ \\
\hline StMADS125 & PGSC0003DMG400026881 & chr11 17707080-17707472 & 130 & 1 & $M \beta$ \\
\hline StMADS126 & PGSC0003DMG400036234 & chr11 29461097-29461483 & 128 & 1 & $M \beta$ \\
\hline StMADS127 & PGSC0003DMG400044576 & chr11 40025123-40025641 & 172 & 1 & $M \beta$ \\
\hline StMADS128 & PGSC0003DMG400044231 & chr12 10345869-10346366 & 165 & 1 & $M \beta$ \\
\hline StMADS129 & PGSC0003DMG400035476 & chr12 10440142-10440801 & 219 & 1 & $M \beta$ \\
\hline StMADS130 & PGSC0003DMG400034608 & chr12 10457590-10458198 & 202 & 1 & $M \beta$ \\
\hline
\end{tabular}


Table 1 The detailed information of potato MADS-box gene family. Genes with an asterisk tail means identified in previous studies (Continued)

\begin{tabular}{|c|c|c|c|c|c|}
\hline Name & Locus & Chromosomal locations & Protein (aa) & Exon & Subfamily \\
\hline StMADS131 & PGSC0003DMG400045649 & chr12 10473059-10473718 & 219 & 1 & $M \beta$ \\
\hline StMADS132 & PGSC0003DMG400044285 & chr12 11631459-11632118 & 219 & 1 & $M \beta$ \\
\hline StMADS133 & PGSC0003DMG400016835 & chr00 15615908-15616303 & 131 & 1 & $M \beta$ \\
\hline StMADS134 & PGSC0003DMG400016838 & chr00 15735051-15735446 & 131 & 1 & $M \beta$ \\
\hline StMADS135 & PGSC0003DMG400005533 & chr00 27564359-27564892 & 177 & 1 & $M \beta$ \\
\hline StMADS136 & PGSC0003DMG400039981 & chr00 27565967-27566461 & 164 & 1 & $M \beta$ \\
\hline StMADS137 & PGSC0003DMG400005534 & chr00 27590544-27591083 & 179 & 1 & $M \beta$ \\
\hline StMADS138 & PGSC0003DMG400036527 & chr01 45487714-45488157 & 147 & 1 & Mү \\
\hline StMADS139 & PGSC0003DMG400024023 & chr01 45617221-45618372 & 383 & 1 & My \\
\hline StMADS140 & PGSC0003DMG400024024 & chr01 45621657-45622640 & 327 & 1 & MY \\
\hline StMADS141 & PGSC0003DMG400039025 & chr03 5616516-5617253 & 245 & 1 & My \\
\hline StMADS142 & PGSC0003DMG400030053 & chr04 20856443-20857615 & 390 & 1 & MY \\
\hline StMADS143 & PGSC0003DMG400034827 & chr04 23826227-23827363 & 378 & 1 & My \\
\hline StMADS144 & PGSC0003DMG400038060 & chr05 38909497-38910506 & 213 & 2 & Mү \\
\hline StMADS145 & PGSC0003DMG400040524 & chr05 39047174-39048184 & 336 & 1 & MY \\
\hline StMADS146 & PGSC0003DMG400045326 & chr05 39101622-39102656 & 344 & 1 & MY \\
\hline StMADS147 & PGSC0003DMG400044997 & chr05 39139534-39142691 & 349 & 2 & My \\
\hline StMADS148 & PGSC0003DMG400044633 & chr05 41389552-41390400 & 282 & 1 & My \\
\hline StMADS149 & PGSC0003DMG400039358 & chr05 $43411054-43411578$ & 174 & 1 & My \\
\hline StMADS150 & PGSC0003DMG400041221 & chr05 $43419172-43421058$ & 259 & 2 & MY \\
\hline StMADS151 & PGSC0003DMG400038225 & chr05 43446588-43447571 & 327 & 1 & My \\
\hline StMADS152 & PGSC0003DMG400040157 & chr07 41201874-41202581 & 235 & 1 & My \\
\hline StMADS153 & PGSC0003DMG400028638 & chr12 50083239-50084024 & 261 & 1 & MY \\
\hline
\end{tabular}

13 and 18 from SQUA group. Moreover, the Ma subfamily members StMADS71-79 located in chr04 were segmental duplicated genes, which shows a cluster in the physical map (Fig. 4b). It clearly shows that there probably a chromosome doubling event in chr04 in the process of potato evolution, which contribute greatly to the expansion of Mo type MADS-box genes.

\section{Phylogenetic relationships and conversed motifs of StMADS proteins}

An unrooted tree was built based on the full-length amino acid sequences of 153 potato, 89 Arabidopsis, and 60 rice MADS-box proteins using MEGA6.0 software (Fig. 1). StMADS proteins can be classified into two major subfamilies, MIKC (also known as type II, 39 genes) and M-type (also known as type I, 114 genes), based on the phylogenetic tree. MIKC subfamily can be further divided into MIKC ${ }^{\mathrm{C}}$ (30 genes) and MIKC* (9 genes), whereas M-type contains $M \alpha$ (70), $M \beta$ (28) and My (16). According to the classification method defined in Malus domestica, Oryza sativa, and Brassica rapa, $\mathrm{MIKC}^{\mathrm{C}}$ subgroup was organized into 13 clades. Interestingly, potato MADS-box genes were absent in the FLC-like, AGL15-like, and TT16-like clades.
Subsequently, potato $\mathrm{MIKC}^{\mathrm{C}}$ subgroup consisted of ten clades. TM3-like clade was the largest clade containing seven StMADS proteins. The orthologous and paralogous relationships of MADS-box proteins are analyzed in potato, rice, and Arabidopsis, it was found that most of the M-type subfamily members were concentrated in a cluster, namely all of these homologous MADS-box proteins are paralogous genes. These results indicated that the MADS-box gene family was formed in an ancestral species before the divergence of monocotyledonous and dicotyledonous plants, which was consistent with the results of previous studies [12, 39-52]. Moreover, orthologous pairs come from the MIKC family were with relatively high homology, indicating that the functions of MIKC family genes were relatively conservative in the evolutionary process.

Similarly, an unrooted tree was also built based on the full-length amino acid sequences of 153 potato MADS-box proteins, which could be partitioned into $\mathrm{MIKC}^{\mathrm{C}}$, MIKC*, $\mathrm{M} \alpha, \mathrm{M} \beta$, and $\mathrm{M} \gamma$ with good supporting values (Fig. 5a). To further analyze the motif compositions of potato MADS-box proteins, MEME online software was used to analyze the conserved motifs (Fig. 5b). The number of conserved motif was set to 20 , where motifs $1,2,3$, and 20 


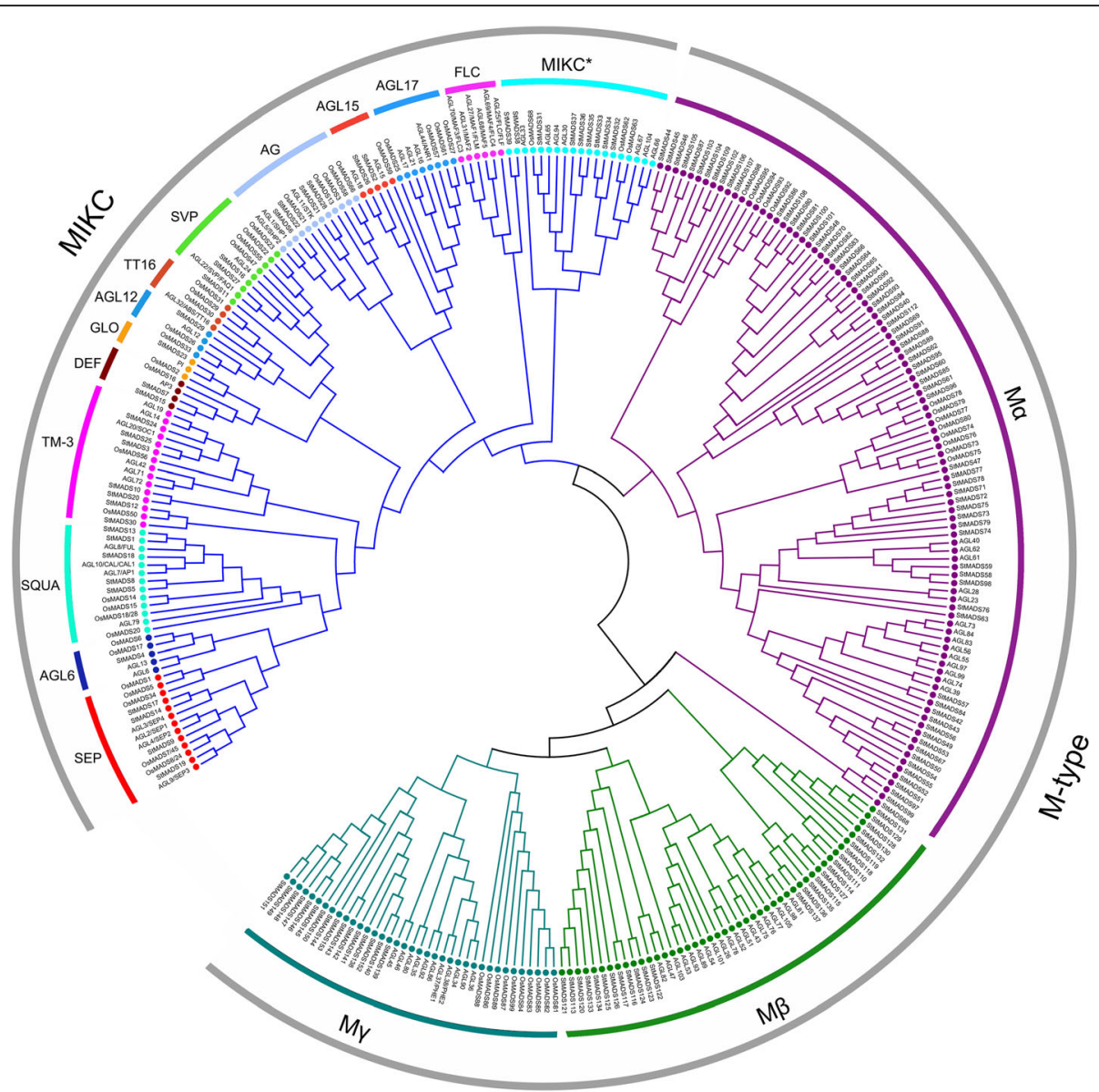

Fig. 1 Phylogenetic tree of Arabidopsis, rice and potato MADS-box proteins. A total of 153 protein sequences of potato MADS-box genes, 89 of rice and 60 of Arabidopsis were pre-aligned by ClustalX (1.83) and used for constructing a NJ-tree in Mega 7 with 1000 replicates in bootstrap values. As is shown above, all clades are colored and arced to make it clear

Table 2 The number of MADS genes in thirteen plant species

\begin{tabular}{|c|c|c|c|c|c|c|c|c|c|}
\hline Species & Genome size (Mb) & Total MADS genes & Total MIKC & $\mathrm{MIKC}^{\mathrm{C}}$ & MIKC ${ }^{*}$ & Total M-type & $\mathrm{Ma}$ & $M \beta$ & $\overline{M y}$ \\
\hline Cucumis sativus & 350 & 43 & 33 & 30 & 3 & 10 & 5 & 2 & 3 \\
\hline Brachypodium distachyon & 260 & 57 & 39 & 32 & 7 & 18 & 9 & 7 & 2 \\
\hline Sesamum indicum & 340 & 57 & 33 & 28 & 5 & 24 & 14 & 0 & 10 \\
\hline Oryza sativa & 466 & 75 & 44 & 38 & 6 & 32 & 13 & 9 & 10 \\
\hline Prunus mume & 280 & 80 & 37 & 32 & 5 & 43 & 20 & 14 & 9 \\
\hline Vitis vinifera & 490 & 90 & 48 & 42 & 6 & 42 & 23 & 0 & 19 \\
\hline Populus trichocarpa & 480 & 105 & 64 & 55 & 9 & 41 & 23 & 12 & 6 \\
\hline Arabidopsis thaliana & 125 & 106 & 45 & 39 & 6 & 61 & 25 & 20 & 16 \\
\hline Solanum lycopersicum & 900 & 107 & 53 & 41 & 12 & 54 & 34 & 6 & 14 \\
\hline Raphanus raphanistrum & 253 & 144 & 76 & 70 & 6 & 68 & 31 & 12 & 25 \\
\hline Malus domestica & 1874 & 146 & 92 & 75 & 17 & 54 & 22 & 8 & 24 \\
\hline Solanum tuberosum & 844 & 153 & 39 & 30 & 9 & 114 & 70 & 28 & 16 \\
\hline Brassica rapa & 485 & 167 & 100 & 89 & 11 & 67 & 29 & 16 & 22 \\
\hline
\end{tabular}




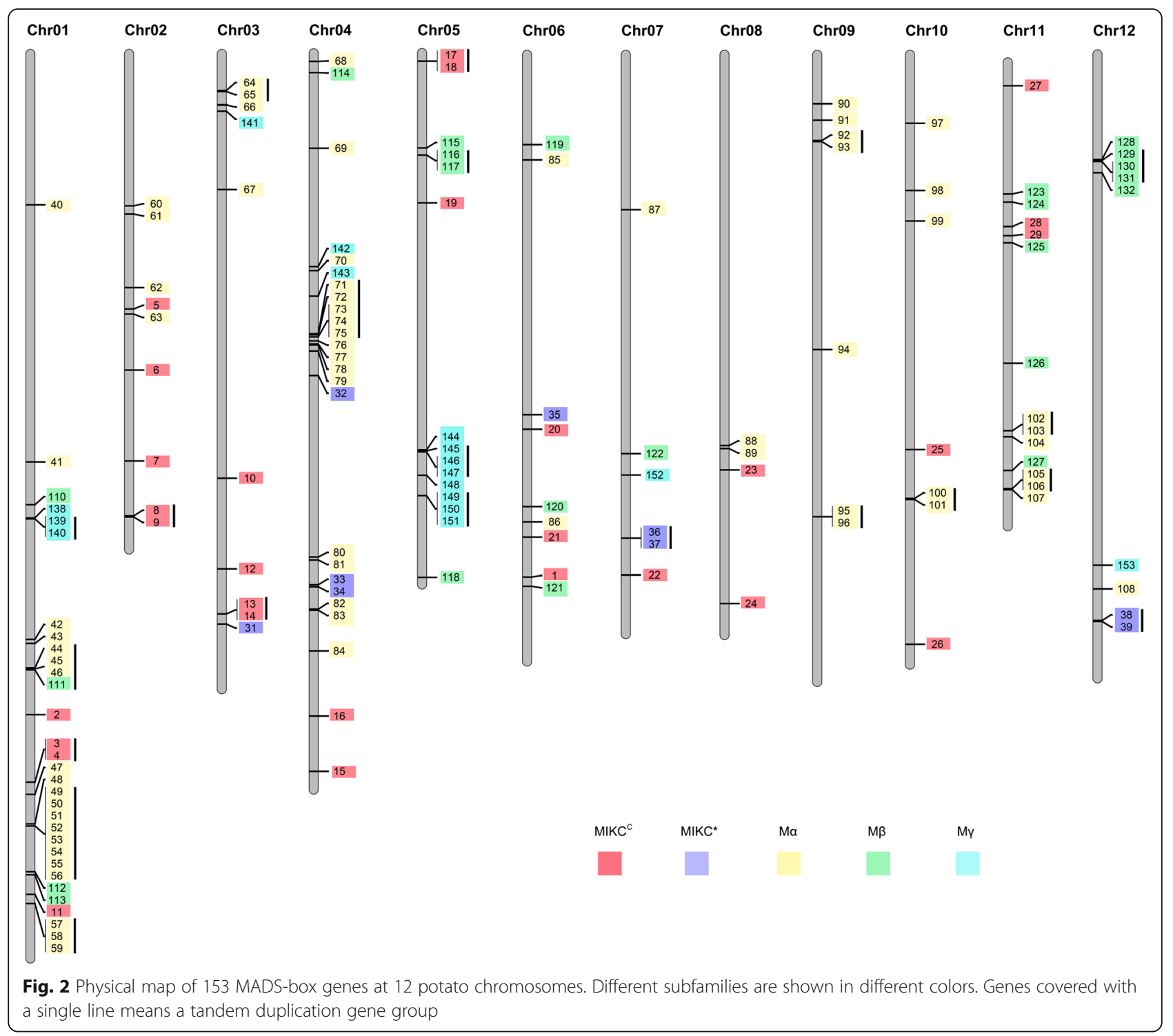

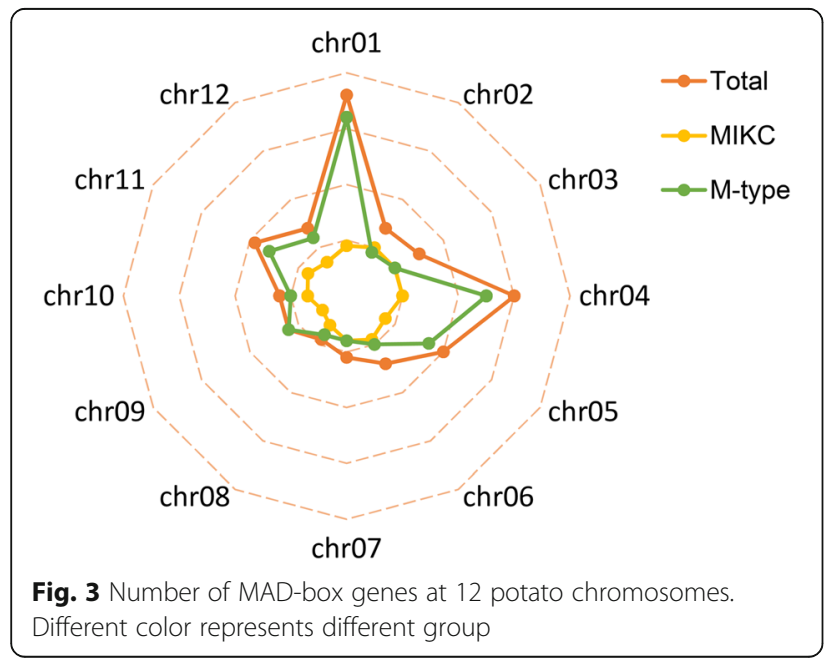

were located at MADS-box domains and motifs 6, 11 and 16 were located at $\mathrm{K}$ domain. Moreover, motifs 4, 5, and 7-10 represented coil regions and low complexity regions. Besides, the rest motifs were less conservative and only appeared in several MADS-box proteins. As shown in Fig. 4, MIKC $^{C}$ subgroup contains seven conservative motifs 1 , $2,3,6,11,16$, and 20 and motifs 11 and 16 belonging to $K$ domain only existed in this subgroup. The MIKC* subgroup contains fewer motif varieties, mainly motifs 1 and 3 , and some of them had motifs 6 and 20 similar to MIKC. Specifically, StMADS31 had motif 18 adjacent to motif6 which was similar to the members belonging to subgroup $\mathrm{M} \alpha$. These results showed that the MADS-box proteins included in the same clade in the phylogenetic tree have almost identical motif distribution types. Moreover, the structure of MIKC* had both characteristics of M-type and $\mathrm{MIKC}^{\mathrm{C}}$, but in Arabidopsis the subgroup MIKC* was 


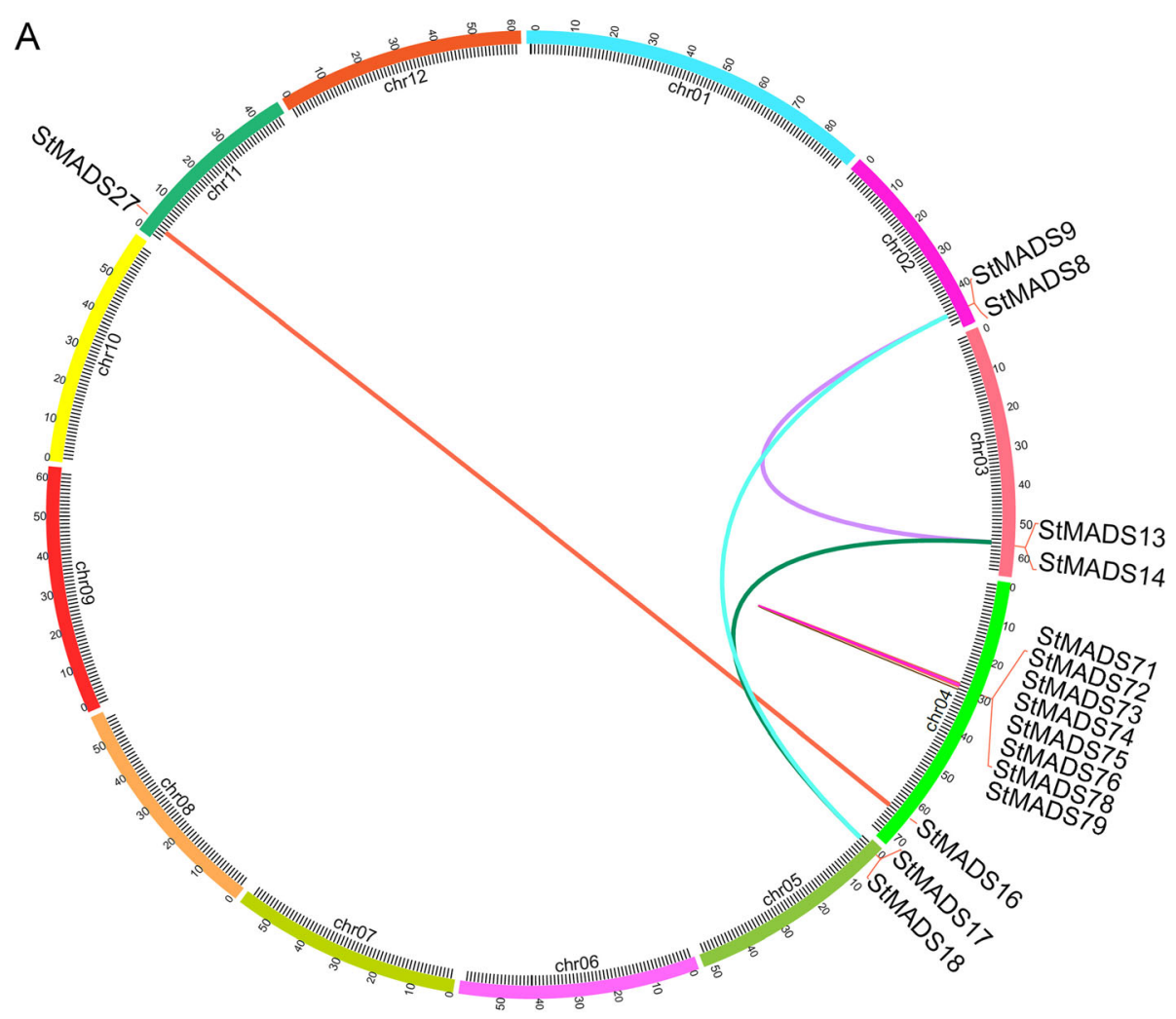

B Syntenic MADS-box genes in Chr04_27499096-28566718

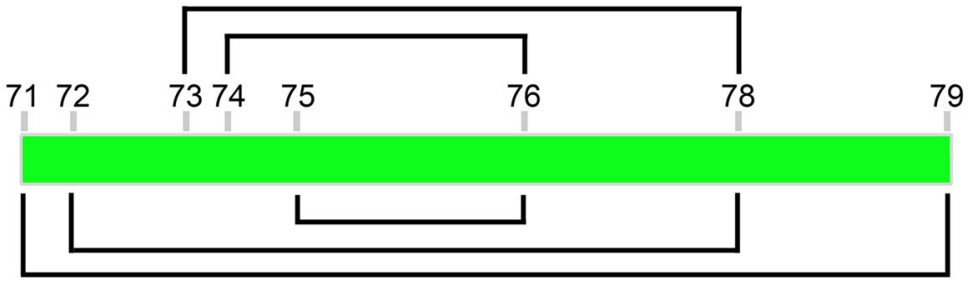

Fig. 4 Segmental duplication genes in 12 potato chromosomes. Genes linked with a line shows a pair of segmental duplicated genes. a Segmental gene pairs between 12 chromosomes. b Micro-syntenic map in Chr04_27,499,096-28,566,718

attributed to $\mathrm{M} \delta$, which were attributed to MIKC group later in the analyses of MADS-box families of other species $[12,39-52]$.

\section{Phylogenetic relationship of MADS-box genes of potato and tomato}

Tomato is the most studied model plant in Solanaceae family. Therefore, a comparison with tomato MADS genes could provide more clues on the function differentiation of potato MADS genes (Fig. 6 and Additional file 2: Table S2). There was a total of 107 MADS-box genes in tomato, including 53 MIKC type and 54 M-type MADS-box genes. Tomato compromised more MIKC genes (53) compared with those in potato (39) even if the total number of potato MADS-box genes (153) is more than those in tomato (107). On the contrary, there were more M-type MADS-box genes in potato compared with those in tomato, especially the number of $M \alpha(70)$ and $M \beta$ (28) in higher than those in tomato $(M \alpha, 34 ; M \beta, 6)$, respectively (Fig. 6 and Table 2). These evidences suggested that the expansion of MADS-box in Solanaceae might be quite different.

To speculate the functions of potato MADS-box genes, we compare the MIKCC MADS-box genes with their closely related homologs. The orthologs of MIKCC potato MADS-box genes was screened by following criteria, which were BLASTP e-Value was less than 10e-10) with more than $80 \%$ coverage in length 


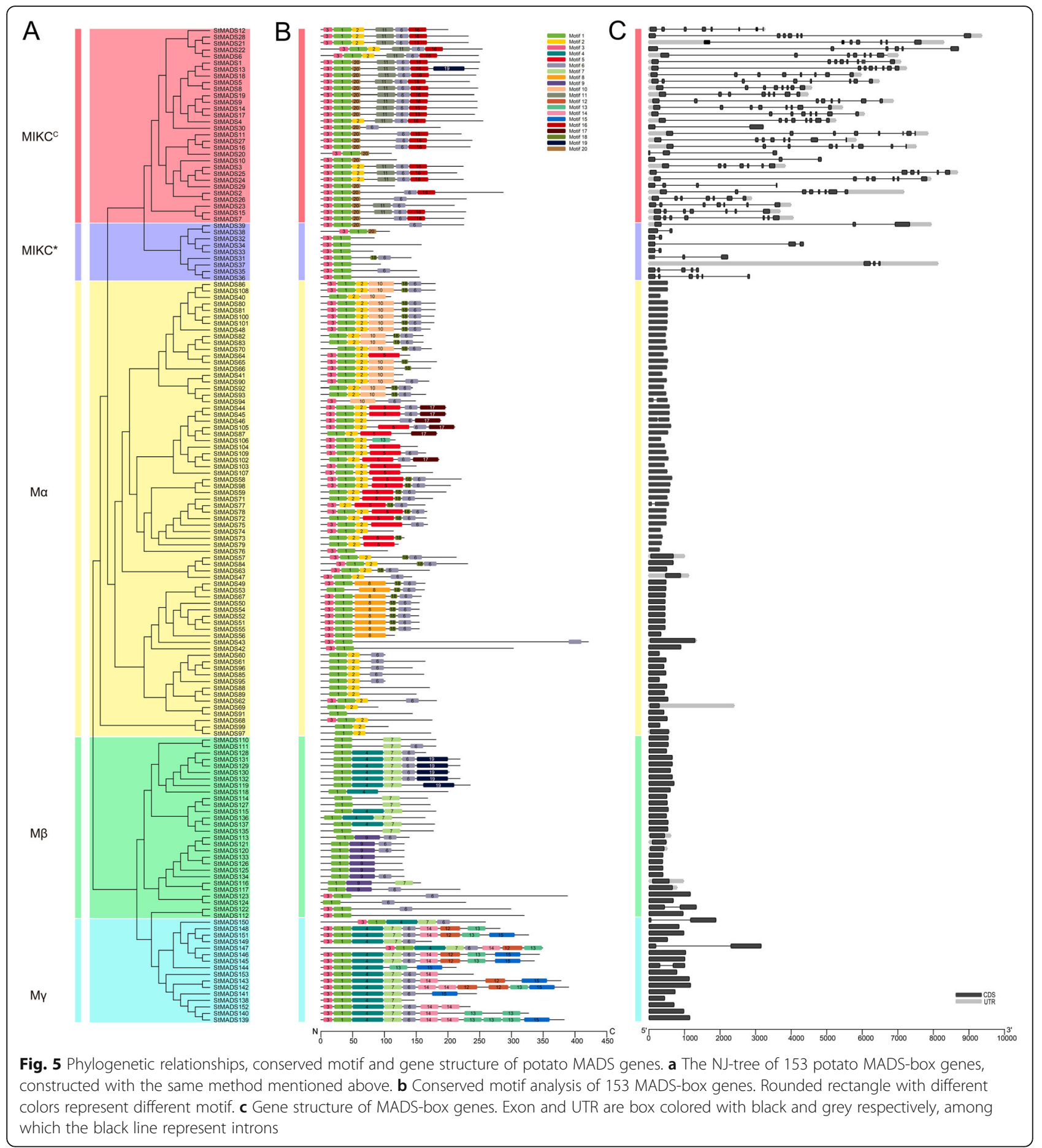

and the ortholog was the best-matching homolog than other candidate in tomato. The orthologs of most potato MIKCC MADS-box genes could be found in tomato except StMADS3, 5, and 20 (Additional file 3: Table S3). The Orthologs in different species have evolved from a common ancestral gene via speciation, which often retain the same functions during evolution.

\section{Tissue specific expression patterns of $\operatorname{MIKC}^{\mathrm{C}}$ StMADS} genes

Illumina RNA-Seq transcriptome data of DM and RH was retrieved to explore the expression patterns of StMADS genes, including vegetative organs (including root, stem, petiole, and leaf), floral organs (including flower, stamen, sepal, petal, and carpel) and storage organs (including stolon and tuber) [36]. The expression 


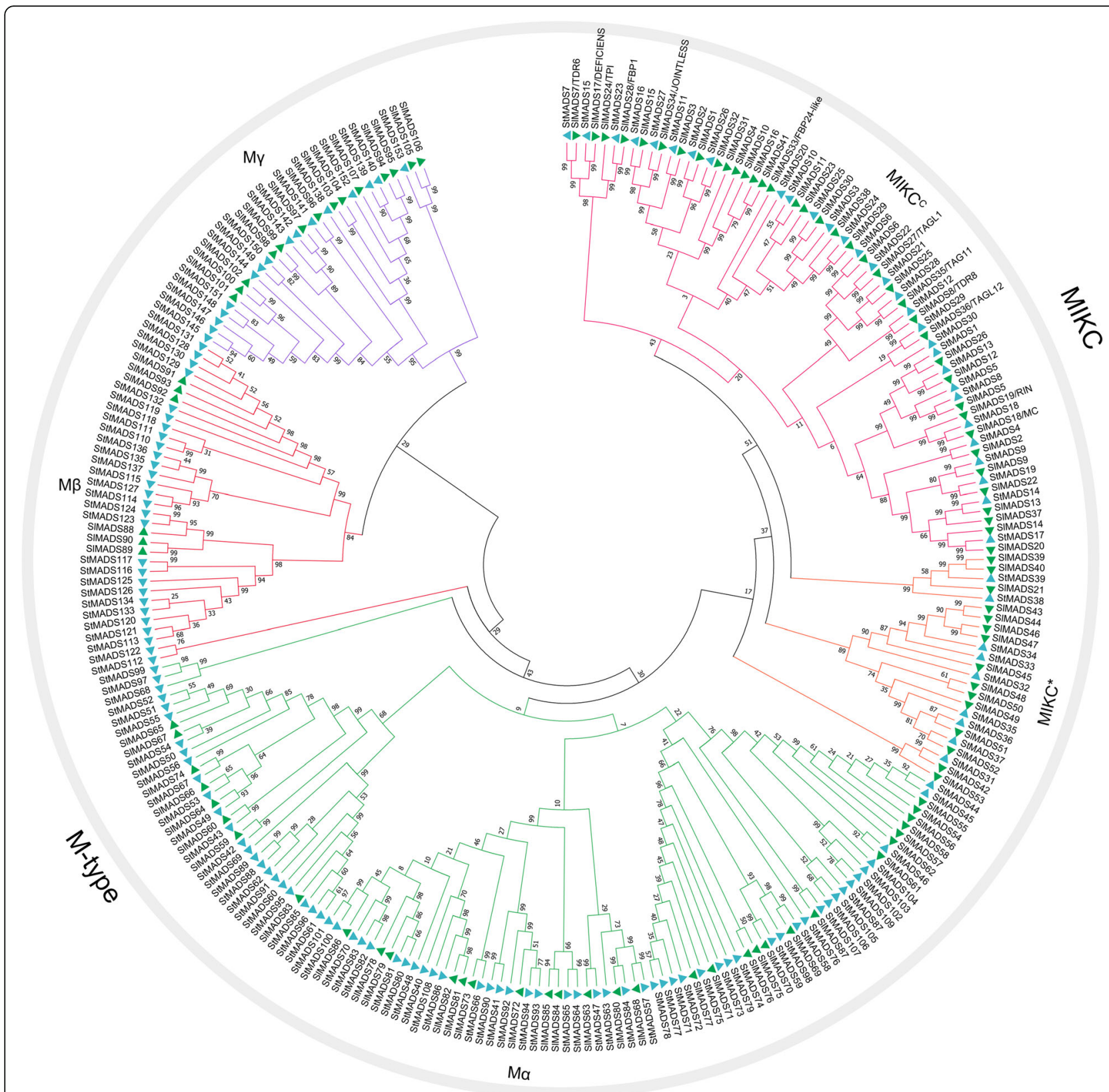

Fig. 6 Phylogenetic analyses of MADS-box genes between Solanum tuberosum and Solanum lycopersicum. The NJ-tree was generated using the method mentioned above

levels of StMADS genes were estimated by using FKPM (fragments per kilobase of transcript per million mapped fragment) method. A gene was designated as expressed if its FKPM value in any tissue or organ was greater than 1. According to this standard, 37 of 39 MIKC-type StMADS genes were expressed but only 16 of 123 M-type StMADS genes were expressed, indicating that MIKC-type StMADS genes were actively expressed and most of M-type StMADS genes were not expressed even if there were many M-type StMADS genes presented on the potato genome.
The MIKC ${ }^{\mathrm{C}}$ StMADS genes were selected for further expression analysis because they were probable downstream targets of tuberigen StSP6A based on previous studies about its homologue-FLOWERING LOCUS $\mathrm{T}$ in Arabidopsis and rice [34]. Hierachical clustering of MIKC ${ }^{\mathrm{C}}$ StMADS genes was performed by using the transcriptome data of DM and RH, respectively (Fig. 7). The MIKC ${ }^{\mathrm{C}}$ StMADS genes in both DM and $\mathrm{RH}$ were similarly divided into two major clusters. The first group of MIKC ${ }^{\mathrm{C}}$ StMADS genes was mainly expressed in floral organs and the other group was expressed in vegetative 

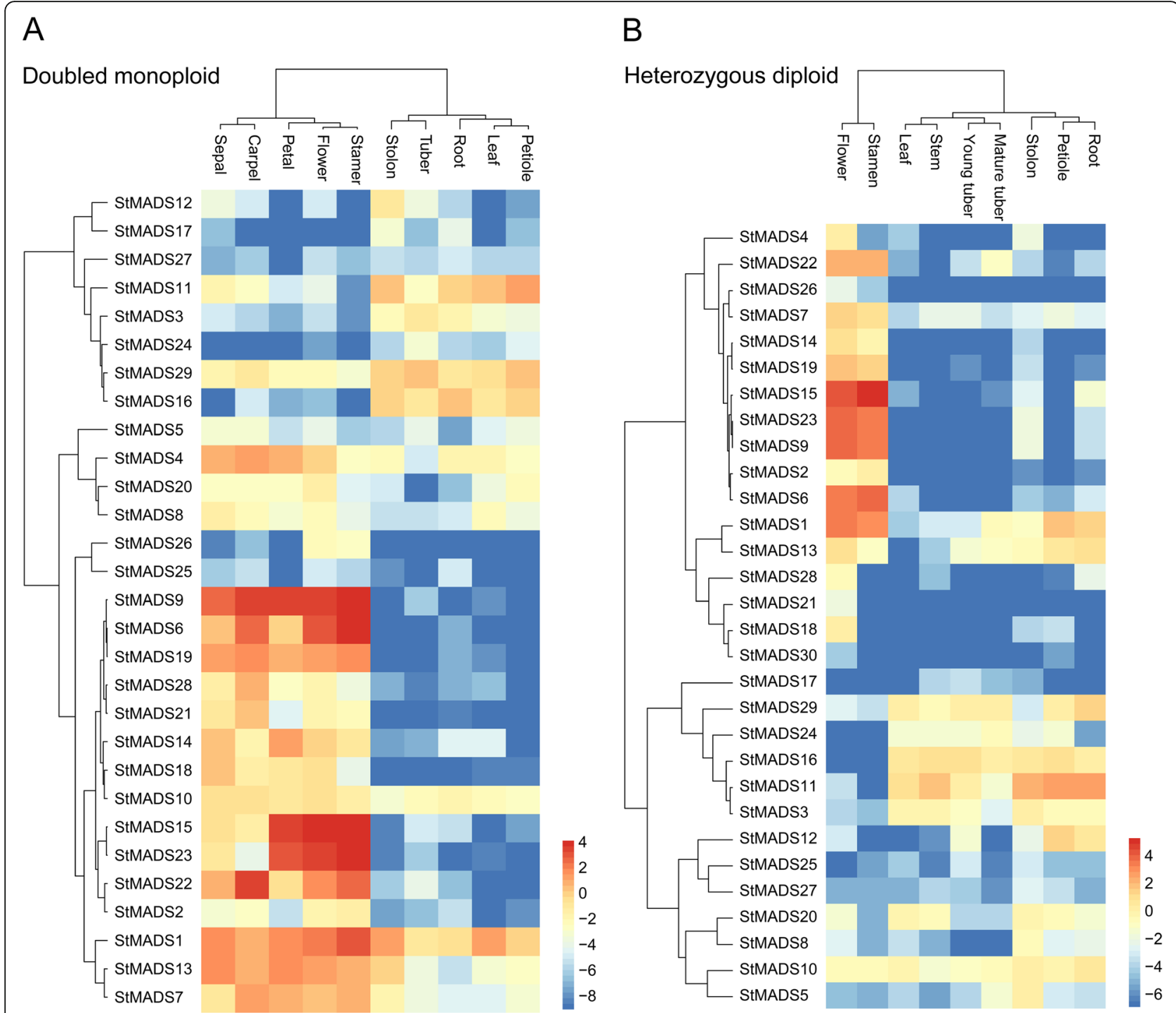

Fig. 7 Expression profiles of MADS-box genes in double monoploid and heterozygous. The RNA-seq data is retrieved from PGSC. FPKM values of MADS genes are normalized and the heatmap is drawn with Pretty Heatmap at ImageGP. a Expression profiles of the StMADS genes in DM (doubled monoploid S. tuberosum Group PhurejaDM1-3). b Expression profiles of the StMADS genes in RH (heterozygous diploid S. tuberosum Group Tuberosum RH89-039-16)

and storage organs. More specifically for their expressions in DM, 21 genes, five genes, and four genes were with highest expression in floral organs, vegetative organs, and storage organs, respectively (Fig. 7a). And for RH, 16 genes, five genes, and nine genes were with highest expression in floral organs, vegetative organs, and storage organs, respectively (Fig. 7b). It was found that most MIKC $^{\mathrm{C}}$ StMADS genes were expressed in floral organs, indicating their possible roles in controlling floral organ development. Whereas, there were more $\mathrm{MIKC}^{\mathrm{C}}$ StMADS genes expressed in storage organs of RH compared with DM.

Moreover, the expressions of MIKC ${ }^{\mathrm{C}}$ StMADS genes in storage organs were further analyzed. It was found that 17 and 26 MIKC $^{\mathrm{C}}$ StMADS genes were expressed (FKPM >1) in storage organs of DM and $\mathrm{RH}$, respectively. And nine and eight and $12 \mathrm{MIKC}^{\mathrm{C}}$ StMADS genes were highly expressed (FKPM >10) in storage organs of DM and $\mathrm{RH}$, respectively. Taken together, six genes (StMADS1, 3, 11, 13, 16, and 29) were consistently with high expression levels in storage organs of both $\mathrm{DM}$ and $\mathrm{RH}$, which may be involved in tuberization and following tuber development. Based on the phylogenic relationship, it was found that StMADS1 and StMADS13 were homologous genes of AGL8/FUL and OsMADS14/15, StMADS3 was homologous gene of SOC1 and OsMADS56, StMADS11 and StMADS16 were homologous genes of AGL22/SVP, and StMADS29 was homologous gene of AGL12. Among these genes expressed in potato stolon and tubers, StMADS1, StMADS3, and StMADS13 were the most likely 
downstream genes of tuberigen StSP6A because their homologous genes AGL8/FUL, OsMADS14/15, and SOC1 were proved to be downstream targets of Arabidopsis and rice FLOWERING LOCUS T [53-57].

\section{QRT-PCR verifications of tissue specific MIKC ${ }^{C}$ StMADS genes}

To validate the results of RNA-seq analysis, real-time PCR analysis was performed for 29 MIKC $^{\mathrm{C}}$ StMADS genes. Our tests showed that the real-time PCR experiments of 25 MIKC $^{\mathrm{C}}$ StMADS genes (except StMADS7, 10, 20, and 26) were successfully conducted in tissues including roots, leaves, stolons, young tubers, mature tubers, and flowers. The results of real-time PCR showed that the expression patterns of most were in general agreement with the data of RNA-seq analysis. For example, eleven StMADS genes (StMADS4, 6, 9, 14, 15, 18, 19, 21, 22, 23, and 28) were overwhelmingly expressed in flowers compared with any other tissues (Fig. 8a), which were perfectly consistent with their expression patterns in flowers of both DM and RH (Fig. 7). The StMADS genes specifically expressed in potato flowers were most likely to control floral organ formation like their homologues in $\mathrm{ABCDE}$ model of other species $[58,59]$.

StMADS1, 12, 13, and 27 were not only expressed in flowers but also expressed in stolons and young tubers (Fig. 8b), indicating that they might control the formations of both flower organs and tuberization. StMADS3, 11,16 , and 17 were highly expressed in stolons and/or young tubers but their expressions in flower were relatively low (Fig. 8c). Besides, we found six StMADS genes were expressed in almost all examined tissues without obvious tissue specific patterns (Fig. 8d, the expressions of StMADS24 and StNADS29 were not showed.

\section{Screening for downstream targets of tuberigen StSP6A}

StSP6A, a FLOWERING LOCUS T homologue in potato, have been reported to be a mobile signal in controlling not only flowering but also tuberization, while its homologue StSP3D is mainly involved in floral transition [34]. According to previous studies about flowering, MADS-box genes encoding proteins involved in flowering identity determination are major targets of FLOWERING LOCUS $\mathrm{T}$ and it was speculated that StMADS genes were downstream targets of tuberigen StSP6A. Therefore, the whole-genome microarray data from stolon tissue of StSP6A-overexpression (StSP6A-OX) and StSP6A-RNAi plants was used to screen for downstream StMADS genes. Firstly, the DNA probe sequences on POCI (Potato Oligo Chip Initiative) [34] microarrays were used as queries to perform BLASTN searches against the transcript sequences of StMADS genes. It was found that 22 probes corresponded to 17 StMADS genes (16 genes were belonged to $M I K C^{C}$ type) were presented on the POCI microarray chip and four genes had two or three probes. Hierachical clustering of the expressions of StMADS genes in StSP6A-OX and StSP6A-RNAi plants showed that StMADS1 and StMADS13 were the most possible downstream targets of StSP6A because the expressions of StMADS1 and StMADS13 were activated in both biological replicates of StSP6A-OX plants and was repressed in both biological replicates of StSP6A-RNAi plants. The expressions of StMADS3 and StMADS11 were only activated in one biological replicate of StSP6A-OX plants and not repressed in StSP6A-RNAi plants. The expressions of other StMADS genes were not obviously changed in either StSP6A-OX or StSP6A-RNAi plants.

Moreover, to verify the whether the expressions of StAMDS1 and StMADS13 were associated with the expression of StSP6A, we investigate the expressions of StSP6A, StMADS1, and StMADS13 in leaves of 30 days after sprouting, leaves of 60 days after sprouting, and young tubers, respectively. It was found that these three genes were not expressed in potato leaves at juvenile stage (30 days after sprouting), whereas StMADS1 and StSP6A were highly expressed in potato leaves at early flowering stage (60 days after sprouting) and young tubers (Fig. 9b). The expression of StMADS13 was not detected in 60d leaves but was observed in young tubers (Fig. 9b). These results indicated that StMADS1 expressions were associated with StSP6A in both 60d leaves and young tubers, whereas StMADS13 was only associated with StSP6A in young tubers. Though both StMADS1 and StMADS13 were putative downstream genes of StSP6A, their regulatory mechanism might be different depending on tissue types.

\section{Discussion}

Potato is one of the major food crops, which feeds millions of people all over the world [60]. As an important tuber crop, the improvement of yield is a key issue to potato breeder in china, owing to its low production far fewer than the global average. Thus, the investigation of molecular mechanism of tuberization and tuber development remains unclear. It will be helpful to identify candidate genes related to tuberization and tuber development, which are the key resources to promote the improvement of yield for both genetic modified crop and traditional breeding. Previous study had shown that FT protein StSP6A functioned as a mobile signal in controlling tuberization under short-day condition [34], and its paralogue StSP3D was involved in day-neutral flowering control. FT was a classical upstream regulator of MADS-box genes in the conservative $\mathrm{ABC}$ model in flower organ identity [59]. Based on clues that we mentioned above, it was reasonable to believe that some potato MADS-box genes were related to tuberization and tuber development.

In this study, a total of 153 members of MADS-box gene family were characterized in potato (Table 1). To 


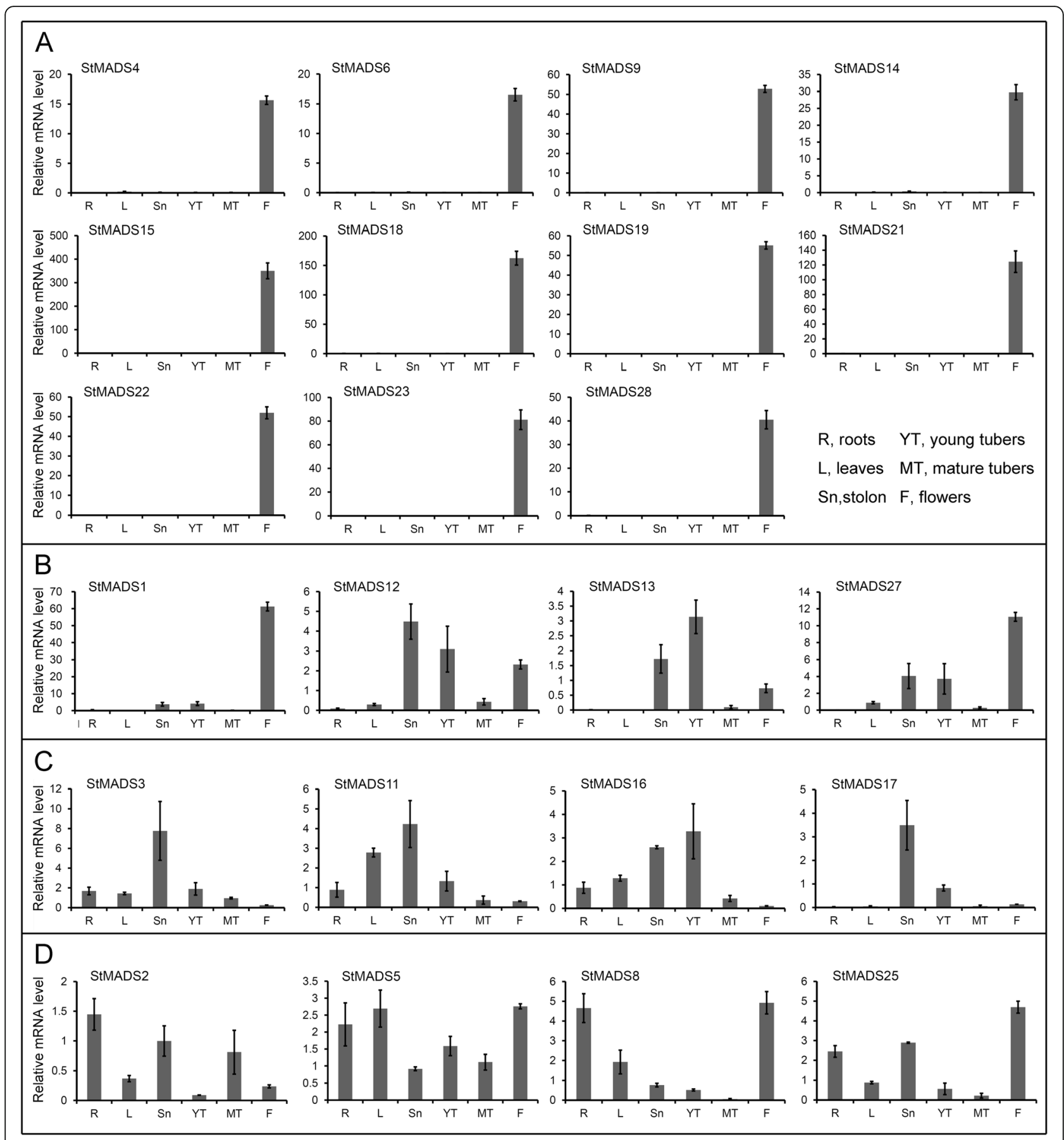

Fig. 8 QRT-PCR verifications of representative StMADS genes in various potato tissues. a Genes are mainly expressed in flowers. b Genes preferentially show expression in flowers, stolons, and young tubers. c Genes are abundantly expressed in stolons. $\mathbf{d}$ Genes are expressed in nearly all examined tissues

confirm the gain and loss of MADS-box genes in potato, a phylogenetic tree was produced using ammo acid sequence of 153 potato MADS-box genes and representative MADS genes of Arabidopsis and rice (Fig. 1). It was found that most of MADS-box genes had their orthologs in potato, except for TT16-like, AGL17-like, and FLC-like subgroups. Besides, it was found that members of M-type, specifically $\mathrm{M} \alpha$, were much more than any other species ever studied (Table 2), implying that potato MADS-box genes might have a different evolution pattern [39-52]. Apparently, the large potato MADS-box family might be due to its larger genome, which was produced by differently genomic duplication events in different species during the course of plant evolution 
A

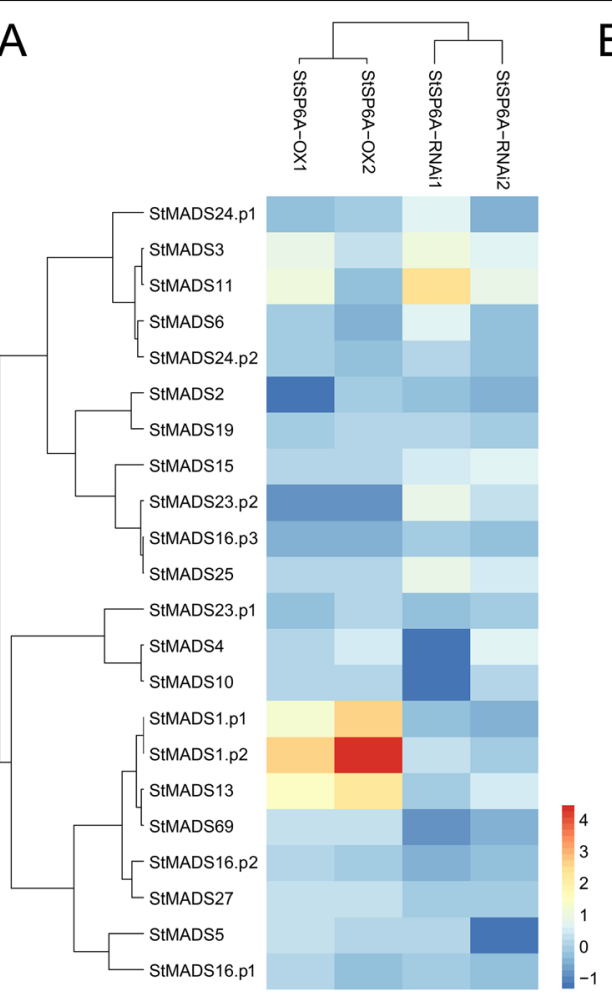

B

$\because$ StSP6A $\quad$ StMADS1 $\because$ StMADS13

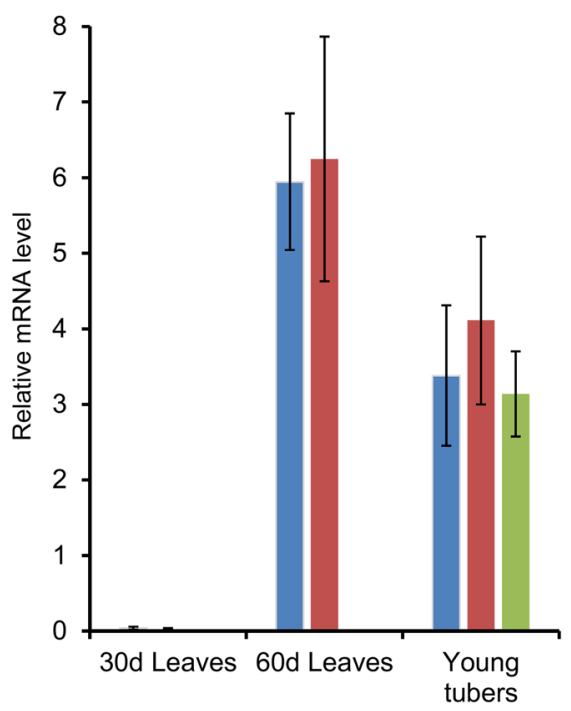

Fig. 9 Expression relationships between MADS-box genes and StSP6A. a Heatmap of the expression of 17 MADS-box genes in StSP6A-OX and StSP6A-RNAi plants. b Expressions of StSP6A, StMADS1, and StMADS13 in leaves of 30 days after sprouting, leaves of 60 days after sprouting, and young tubers, respectively

$[61,62]$. To explore what behind the extremely different composition of MADS-box family in potato, the effects of gene duplication events on expansion of MADS-box genes were investigated (Fig. 2). It was suggested that segmental duplications mainly contributed to expansion of MIKC subfamily, whereas the boom of M-type was mostly derived from tandem duplications (Figs. 2 and 3). It was believed that this phenomenon was due to the M-type genes mainly derived from the site-specific duplications within the same chromosome, while MIKC mainly came from the whole genome duplication events [61, 63-65]. Birth-and-death rates of MADS-box genes after gene duplications in different species were different which resulted in variable number of MADS-box genes in the same subfamily of different species $[61,62]$. These evidences could be one explanation for the abnormality of number of $\mathrm{M} \alpha$ in potato.

Besides gene duplication events, gene mutation and loss of certain domain, might also play important role in generating a part of $M \alpha$ in potato. It had been reported that MIKC" was the intermediate form between MIKC ${ }^{\mathrm{C}}$ and M-type in the course of plant evolution [58, 59, 62]. Based on intron-exon structures of potato MADS-box genes, it was found that the intron number of $\mathrm{MIKC}^{\mathrm{C}}$ was the most and MIKC* had less intron than $\mathrm{MIKC}^{\mathrm{C}}$, whereas most of M-type MADS-box genes was intronless. It could be speculated that the exon-intron loss mutations mainly happened in the K-box domain of $\mathrm{MIKC}^{\mathrm{C}}$ in the process of plant evolution, thus a new group, MIKC", was born. MIKC $C^{*}$ further lost several exons and introns corresponding to K-box domain and then produced M-type, which was also found in previous studies [49]. These evidences could be the other explanation for the abnormality of number of $\mathrm{M} \alpha$ in potato.

To compare the composition of MADS-box genes of potato and tomato, phylogenetic analysis was performed. Since these two species are genetically close species, it was surprising that tomato contain much more MIKC genes, while potato included more M-type genes. This suggested that these species might undergo a different evolution history of MADS-box. The candidate genes StMADS1 and StMADS13 that may be related to tuberization, the functions of their orthologous genes in tomato, SIMADS26 and SIMADS12, were still undiscovered. Though potato and tomato are both Solanaceae plants, the stolons and tubers are only found in potato. Thus, the functions of StMADS1 and StMADS13 need to be investigated in potato. Nevertheless, there was a homolog of StMADS1 and StMADS13, RIN found in tomato, which is proved to play important roles in induction of tomato ripening [66, 67]. Besides, compared with potato, most genes that had been studied in tomato have few homologs, indicating that a 
different history of gain and loss functions of MADS-box genes [68-70].To investigate the possible roles of StMADS genes in tuberization, we used RNA-seq data of DH and RM available in PGSC. Most of the StMADS genes showed tissue-specific expression patterns. Among these genes, StMADS1, 3, 11, 13, 16, and 29 were highly expressed in storage organs of both DM and RH (Fig. 7). Consistent with RNA-seq data, the results of QRT-PCR showed that StMADS1, 3, 11 and 16 were overwhelmingly expressed in stolons and/ or young tubers (Fig. 8), indicating that these genes were probably involved in tuberization and/or tuber development. Previous studies had shown us a fine regulation map of MADS-box genes and its significant roles in flower organ differentiation in several model plants. In Arabidopsis, the expression of FLOWERING LOCUS $T(F T)$, a core flower development regulator, was suppressed by the FLOWERING LOCUS C (FLC), a typical MADS-box genes, bound in its CArG site between first intron and promoter [71-73]. Interestingly, the expressions of MADS-box genes including APETALA1 (AP1) and SUPPRESSOR OF OVEREXPRESSION OF CO 1(SOC1) were related to flowering promotion that was controlled by two interacted flowering-related proteins FT and FD (FLOWERING LOCUS D) [74]. In monocot plants, orthologs and paralogs of FT and MADS-box presented nearly the same transcriptional regulation, for instance, a pair of FT genes Heading-date $3 a$ (Hd3a) and RICE FLOWERING LOCUS T (RFT1) upregulated the expression of OSMADS15, which is crucial for floral initiation [75-78]. Given the highly conservative model of FT and MADS-box genes, it was reasonable to believe that this model would probably work in potato. Recent study showed that there was a functional diversification of FT proteins in potato. StSP3D was mainly involved in floral transition, and StSP6A was involved in tuberization transition. [34]. Therefore, these StMADS genes (StMADS1, 3, 11-13, 17 , and 27) mainly expressed in stolons and/or young tubers were possible downstream targets of StSP6A. Interestingly, it was found StMADS1 and 13 were strongly correlated with the expression of StSP6A in leaves and/or young tubers. More evidences were obtained through analyzing the microarray data from stolon tissue of StSP6A-overexpression (StSP6A-OX) and StSP6A-RNAi plants, the expression of StMADS1 and 13 were upregulated in StSP6A-OX plants and downregulated in StSP6A-RNAi plants. Given the evidence that discussed above, StSP6A and several MADS-box genes are probably share the same regulation map with their homologs in the model plant. However, the truth of how StSP6A regulate StMADS1 and 13, in a directly interaction way or in the promotor region, remain unclear. As a master of transcription, tracing the target of
MADS-box gene would also be a valuable subject in the future study.

\section{Conclusion}

This study present genomic annotation and expression profiling of potato MADS-box genes. Comprehensive analyses about the evolution and functional differentiation of potato MADS-box were also performed, which would provide solid basis for further functional studies about this gene family. Potato MADS-box genes were putative downstream targets of the potato FT homolog tuberigen StSP6A, which is a mobile signal in controlling tuberization. Furthermore, StMADS1 and StMADS13 were believed to be candidate genes in the downstream of StSP6A. Thus, the utilization of these MADS-box genes for both genetically modified crop and traditional breeding practice in genetic improvement would be possible.

\section{Additional files}

Additional file 1: Table S1. QRT-PCR primers corresponding to potato MADS-box genes. (DOCX $18 \mathrm{~kb}$ )

Additional file 2: Table S2. MADS-box genes in tomato. (DOCX 25 kb) Additional file 3: Table S3. Orthologs of potato MIKC ${ }^{C}$ MADS-box genes in tomato. (DOCX $18 \mathrm{~kb}$ )

\section{Abbreviations}

AA: Amino acid; AP1: APETALA1; BLASTP: Basic local alignment search toolprotein; FLC: FLOWERING LOCUS C; FT: FLOWERING LOCUS T;

Hd3a: Heading-date 3a; ORF: Open reading frame; PGDD: Plant genome duplication database; PGSC: Potato genome sequencing consortium; QRTPCR: Quantitative real-time polymerase chain reaction; RFT1: RICE FLOWERING LOCUS T; SOC1: SUPPRESSOR OF OVEREXPRESSION OF CO 1

\section{Acknowledgements}

The authors acknowledge the members of Dr. Chen's lab in State Key Laboratory of Crop Stress Biology in Arid Areas, China for technical laboratory advices.

\section{Funding}

This research was supported by the National Natural Science Foundation of China (31500159), the Natural Science Foundation of Shaanxi Province (2015JQ3090). The funders had no role in study design, data collection and analysis, decision to publish, or preparation of the manuscript.

\section{Availability of data and materials}

All of the datasets supporting the results of this article are included within the article and its Additional files. The raw data generated in this study can be obtained by reasonable request to the corresponding authors.

\section{Authors' contributions}

$\mathrm{HM}$ secured most funding to support this study. $\mathrm{HZ}$ and $\mathrm{HM}$ conceived of and designed the research plans. HG, SL, MH, YZ (Yao Zhou), and YZ (Yaqi Zhao) isolated RNA from potato tissues, conducted the GRT-PCR analysis, and completed statistical analysis of the qRT-PCR analysis dataset. HG, ZW, and GL completed the data processing, normalization, and bioinformatics analyses. HG and $\mathrm{HZ}$ wrote the article with contributions from all the authors. HM supervised and supported the writing. All authors read and approved the final manuscript.

Ethics approval and consent to participate Not applicable. 


\section{Consent for publication}

Not applicable.

\section{Competing interests}

The authors declare that they have no competing interests.

\section{Publisher's Note}

Springer Nature remains neutral with regard to jurisdictional claims in published maps and institutional affiliations.

\section{Author details}

${ }^{1}$ College of Agronomy, Northwest A\&F University, Yangling 712100, Shaanxi, China. ${ }^{2}$ School of Stomatology, Wuhan University, Wuhan 430072, Hubei, China.

Received: 27 April 2018 Accepted: 25 September 2018

\section{Published online: 03 October 2018}

\section{References}

1. Riechmann $J$, Meyerowitz EM. MADS domain proteins in plant development. Biol Chem. 1997;378:1079-101.

2. de Folter $\mathrm{S}$, Angenent GC. trans meets cis in MADS science. Trends Plant Sci. 2006;11:224-31.

3. Gramzow L, Ritz MS, Theißen G. On the origin of MADS-domain transcription factors. Trends Genet. 2010;26:149-53.

4. Passmore S, Maine GT, Elble R, Christ C, Tye BK. Saccharomyces cerevisiae protein involved in plasmid maintenance is necessary for mating of MATa cells. J Mol Biol. 1988;204:593-606.

5. Norman C, Runswick M, Pollock R, Treisman R, Almendral JM, Sommer D, et al. Isolation and properties of cDNA clones encoding SRF, a transcription factor that binds to the c-fos serum response element. Cell. 1988;55:989-1003.

6. Sommer H, Beltrán JP, Huijser P, Pape H, Lönnig WE, Saedler H, et al. Deficiens, a homeotic gene involved in the control of flower morphogenesis in Antirrhinum majus: the protein shows homology to transcription factors. EMBO J. 1990;9:605-13.

7. Yanofsky MF, Ma H, Bowman JL, Drews GN, Feldmann KA, Meyerowitz EM The protein encoded by the Arabidopsis homeotic gene agamous resembles transcription factors. Nature. 1990;346:35-9.

8. West AG, Shore P, Sharrocks AD. DNA binding by MADS-box transcription factors: a molecular mechanism for differential DNA bending. Mol Cell Biol. 1997:17:2876-87.

9. Rounsley SD, Ditta GS, Yanofsky MF. Diverse roles for MADS box genes in Arabidopsis development. Plant Cell. 1995:7:1259-69.

10. Alvarez-Buylla ER, Liljegren SJ, Pelaz S, Gold SE, Burgeff C, Ditta GS, et al. MADS-box gene evolution beyond flowers: expression in pollen, endosperm, guard cells, roots and trichomes. Plant J. 2000;24:457-66.

11. Martinez-Castilla LP, Alvarez-Buylla ER. Adaptive evolution in the Arabidopsis MADS-box gene family inferred from its complete resolved phylogeny. Proc Natl Acad Sci. 2003;100:13407-12

12. Parenicova L. Molecular and phylogenetic analyses of the complete MADSbox transcription factor family in Arabidopsis: new openings to the MADS world. Plant Cell. 2003:15:1538-51.

13. Masiero S, Colombo L, Grini PE, Schnittger A, Kater MM. The emerging importance of type I MADS box transcription factors for plant reproduction. Plant Cell. 2011;23:865-72.

14. Nam J, Kim J, Lee S, An G, Ma H, Nei M. Type I MADS-box genes have experienced faster birth-and-death evolution than type II MADS-box genes in angiosperms. Proc Natl Acad Sci. 2004:101:1910-5.

15. Alvarez-Buylla ER, Pelaz S, Liljegren SJ, Gold SE, Burgeff C, Ditta GS, et al. An ancestral MADS-box gene duplication occurred before the divergence of plants and animals. Proc Natl Acad Sci. 2000;97:5328-33.

16. Masiero S, Imbriano C, Ravasio F, Favaro R, Pelucchi N, Gorla MS, et al. Ternary complex formation between MADS-box transcription factors and the histone fold protein NF-YB. J Biol Chem. 2002;277:26429-35.

17. Kramer EM, Su HJ, Wu CC, Hu JM. A simplified explanation for the frameshift mutation that created a novel C-terminal motif in the APETALA3 gene lineage. BMC Evol Biol. 2006:6:30.

18. Henschel K, Kofuji R, Hasebe M, Saedler H, Münster T, Theißen G. Two ancient classes of MIKC-type MADS-box genes are present in the moss Physcomitrella patens. Mol Biol Evol. 2002;19:801-14.
19. Kaufmann K, Melzer R, Theißen G. MIKC-type MADS-domain proteins: structural modularity, protein interactions and network evolution in land plants. Gene. 2005;347:183-98.

20. Becker A, Theißen G. The major clades of MADS-box genes and their role in the development and evolution of flowering plants. Mol Phylogenet Evol. 2003:29:464-89.

21. Colombo M, Masiero S, Vanzulli S, Lardelli P, Kater MM, Colombo L. AGL23, a type I MADS-box gene that controls female gametophyte and embryo development in Arabidopsis. Plant J. 2008;54:1037-48.

22. Kwantes M, Liebsch D, Verelst W. How MIKC* MADS-box genes originated and evidence for their conserved function throughout the evolution of vascular plant gametophytes. Mol Biol Evol. 2012;29:293-302.

23. Liu Y, Cui S, Wu F, Yan S, Lin X, Du X, et al. Functional conservation of MIKC*-type MADS box genes in Arabidopsis and Rice pollen maturation. Plant Cell. 2013:25:1288-303.

24. Sang X, Li Y, Luo Z, Ren D, Fang L, Wang N, et al. CHIMERIC FLORAL ORGANS1, encoding a monocot-specific MADS box protein, regulates floral organ identity in Rice. Plant Physiol. 2012;160:788-807.

25. Causier B, Schwarz-Sommer Z, Davies B. Floral organ identity: 20 years of ABCs. Semin Cell Dev Biol. 2010;21:73-9.

26. Causier B, Castillo R, Xue Y, Schwarz-Sommer Z, Davies B. Tracing the evolution of the floral homeotic B-and C-function genes through genome synteny. Mol Biol Evol. 2010;27:2651-64.

27. Guo J, Shi XX, Zhang JS, Duan YH, Bai PF, Guan XN, et al. A type I MADSbox gene is differentially expressed in wheat in response to infection by the stripe rust fungus. Biol Plantarum. 2013;57:540-6.

28. Adamczyk BJ, Fernandez DE. MIKC* MADS domain heterodimers are required for pollen maturation and tube growth in Arabidopsis. Plant Physiol. 2009;149:1713-23.

29. García-Maroto F, Ortega N, Lozano R, Carmona MJ. Characterization of the potato MADS-box gene STMADS16 and expression analysis in tobacco transgenic plants. Plant Mol Biol. 2000:42:499-513.

30. Kang S-G, Hannapel DJ, Suh S-G. Potato MADS-box gene POTM1-1 transcripts are temporally and spatially distributed in floral organs and vegetative meristems. Mol Cells. 2003;15:48-54.

31. Kan S, Kan H. Characterization of potato vegetative MADS. Box gene, POTML-1 , in response to hormone applications. J Plant Biol. 2002;45:196-200.

32. Rosin FM. Suppression of a vegetative MADS box gene of potato activates axillary meristem development. Plant Physiol. 2003;131:1613-22.

33. Carmona MJ, Ortega N, Garcia-Maroto F. Isolation and molecular characterization of a new vegetative MADS-box gene from Solanum tuberosum L. Planta. 1998;207:181-8.

34. Navarro C, Abelenda JA, Cruz-Oró E, Cuéllar CA, Tamaki S, Silva J, et al. Control of flowering and storage organ formation in potato by FLOWERING LOCUS T. Nature. 2011:478:119-22.

35. González-Schain ND, Díaz-Mendoza M, Zurczak M, Suárez-López P. Potato CONSTANS is involved in photoperiodic tuberization in a graft-transmissible manner. Plant J. 2012;70:678-90.

36. Xu X, Pan S, Cheng S, Zhang B, Mu D, Ni P, et al. Genome sequence and analysis of the tuber crop potato. Nature. 2011;475:189-95.

37. Voorrips RE. MapChart: software for the graphical presentation of linkage maps and QTLs. J Hered. 2002;93:77-8.

38. Tang X, Zhang N, Si H, Calderón-Urrea A. Selection and validation of reference genes for RT-qPCR analysis in potato under abiotic stress. Plant Methods. 2017:13:85.

39. Leseberg CH, Li A, Kang H, Duvall M, Mao L. Genome-wide analysis of the MADS-box gene family in Populus trichocarpa. Gene. 2006:378:84-94.

40. Arora R, Agarwal P, Ray S, Singh AK, Singh VP, Tyagi AK, et al. MADS-box gene family in rice: genome-wide identification, organization and expression profiling during reproductive development and stress. BMC Genomics. 2007;8:242.

41. Hu L, Liu S, Somers DJ. Genome-wide analysis of the MADS-box gene family in cucumber. Genome. 2012;55:245-56.

42. Cao J, Han X, Zhang T, Yang Y, Huang J, Hu X. Genome-wide and molecular evolution analysis of the subtilase gene family in Vitis vinifera. BMC Genomics. 2014:15:1116.

43. Wei B, Zhang RZ, Guo JJ, Liu DM, Li AL, Fan RC, et al. Genome-wide analysis of the MADS-box gene family in Brachypodium distachyon. PLoS One. 2014; 9:e84781.

44. Saha G, Park J-I, Jung H-J, Ahmed NU, Kayum MA, Chung M-Y, et al. Genome-wide identification and characterization of MADS-box family genes 
related to organ development and stress resistance in Brassica rapa. BMC Genomics. 2015;16:178

45. Li C, Wang Y, Xu L, Nie S, Chen Y, Liang D, et al. Genome-wide characterization of the MADS-box gene family in radish (Raphanus sativus L.) and assessment of its roles in flowering and floral organogenesis. Front Plant Sci. 2016;7:1390.

46. Duan W, Song X, Liu T, Huang Z, Ren J, Hou X, et al. Genome-wide analysis of the MADS-box gene family in Brassica rapa (Chinese cabbage). Mol Gen Genomics. 2014;290:239-55.

47. Xu Z, Zhang Q, Sun L, Du D, Cheng T, Pan H, et al. Genome-wide identification, characterisation and expression analysis of the MADS-box gene family in Prunus mume. Mol Gen Genomics. 2014;289:903-20.

48. Tian Y, Dong Q, Ji Z, Chi F, Cong P, Zhou Z. Genome-wide identification and analysis of the MADS-box gene family in apple. Gene. 2015;555:277-90.

49. Wei X, Wang L, Yu J, Zhang Y, Li D, Zhang X. Genome-wide identification and analysis of the MADS-box gene family in sesame. Gene. 2015;569:66-76.

50. Fan CM, Wang X, Wang YW, Hu RB, Zhang XM, Chen JX, et al. Genomewide expression analysis of soybean MADS genes showing potential function in the seed development. PLoS One. 2013;8:e62288.

51. Shu Y, Yu D, Wang D, Guo D, Guo C. Genome-wide survey and expression analysis of the MADS-box gene family in soybean. Mol Biol Rep. 2013;40:3901-11.

52. Grimplet J, Martínez-Zapater JM, Carmona MJ. Structural and functional annotation of the MADS-box transcription factor family in grapevine. BMC Genomics. 2016:17:80.

53. Ferrándiz C, Liljegren SJ, Yanofsky MF. Negative regulation of the SHATTERPROOF genes by FRUITFULL during Arabidopsis fruit development. Science (80- ). 2000;289:436-8.

54. Lim J, Moon YH, An G, Jang SK. Two rice MADS domain proteins interact with OsMADS1. Plant Mol Biol. 2000:44:513-27.

55. Yoo SK. CONSTANS activates SUPPRESSOR OF OVEREXPRESSION OF CONSTANS 1 through FLOWERING LOCUS T to promote flowering in Arabidopsis. Plant Physiol. 2005;139:770-8.

56. Kim SL, Lee S, Kim HJ, Nam HG, An G. OsMADS51 is a short-day flowering promoter that functions upstream of Ehd1, OsMADS14, and Hd3a. Plant Physiol. 2007;145:1484-94.

57. Liu C, Chen H, Er HL, Soo HM, Kumar PP, Han J-H, et al. Direct interaction of AGL24 and SOC1 integrates flowering signals in Arabidopsis. Development. 2008;135:1481-91.

58. Kanno A, Saeki H, Kameya T, Saedler H, Theissen G. Heterotopic expression of class B floral homeotic genes supports a modified ABC model for tulip (Tulipa gesneriana). Plant Mol Biol. 2003;52:831-41.

59. Litt A, Kramer EM. The ABC model and the diversification of floral organ identity. Semin Cell Dev Biol. 2010;21:129-37.

60. Birch PRJ, Bryan G, Fenton B, Gilroy EM, Hein I, Jones JT, et al. Crops that feed the world 8: potato: are the trends of increased global production sustainable? Food Secur. 2012:4:477-508.

61. Airoldi CA, Davies B. Gene duplication and the evolution of plant MADS-box transcription factors. J Genet Genomics. 2012;39:157-65.

62. Nam J, DePamphilis CW, Ma H, Nei M. Antiquity and evolution of the MADS-box gene family controlling flower development in plants. Mol Biol Evol. 2003;20:1435-47.

63. Pinyopich A, Ditta GS, Savidge B, Liljegren SJ, Baumann E, Wisman E, et al. Assessing the redundancy of MADS-box genes during carpel and ovule development. Nature. 2003;424:85-8.

64. Edger PP, Pires JC. Gene and genome duplications: the impact of dosagesensitivity on the fate of nuclear genes. Chromosom Res. 2009;17:699-717.

65. Gramzow L, Weilandt L, Theißen G. MADS goes genomic in conifers: towards determining the ancestral set of MADS-box genes in seed plants. Ann Bot. 2014;114:1407-29.

66. Ito Y, Nishizawa-Yokoi A, Endo M, Mikami M, Shima Y, Nakamura N, KotakeNara E, Kawasaki S, Toki S. Re-evaluation of the rin mutation and the role of RIN in the induction of tomato ripening. Nat Plants. 2017:3:866-74.

67. Vrebalov J, Ruezinsky D, Padmanabhan V, et al. A MADS-box gene necessary for fruit ripening at the tomato ripening-inhibitor (Rin) Locus. Science. 2002; 296:343-6.

68. Quinet M, Bataille G, Dobrev PI, Capel C, Gomez P, Capel J, Lutts S, Motyka V, Angosto T, Lozano R. Transcriptional and hormonal regulation of petal and stamen development by STAMENLESS, the tomato (Solanum lycopersicum L.) orthologue to the B-class APETALA3 gene. J Exp Bot. 2014;65:2243-56.

69. Wang S, Lu G, Hou Z, Luo Z, Wang T, Li H, Zhang J, Ye Z. Members of the tomato FRUITFULL MADS-box family regulate style abscission and fruit ripening. J Exp Bot. 2014;65:3005-14.
70. Nakano T, Kimbara J, Fujisawa M, Kitagawa M, Ihashi N, Maeda H, Kasumi T, Ito Y. MACROCALYX and JOINTLESS interact in the transcriptional regulation of tomato fruit abscission zone development. Plant Physiol. 2012;158:439-50.

71. Helliwell CA, Wood CC, Robertson M, James Peacock W, Dennis ES. The Arabidopsis FLC protein interacts directly in vivo with SOC1 and FT chromatin and is part of a high-molecular-weight protein complex. Plant $\mathrm{J}$. 2006;46:183-92.

72. Lee H, Suh SS, Park E, Cho E, Ahn JH, Kim SG, et al. The AGAMOUS-IIKE 20 MADS domain protein integrates floral inductive pathways in Arabidopsis. Genes Dev. 2000;14:2366-76.

73. Michaels SD. Flowering time regulation produces much fruit. Curr Opin Plant Biol. 2009;12:75-80

74. Srikanth A, Schmid M. Regulation of flowering time: all roads lead to Rome. Cell Mol Life Sci. 2011;68:2013-37.

75. Chen M, Ni M. RFI2, a RING-domain zinc finger protein, negatively regulates CONSTANS expression and photoperiodic flowering. Plant J. 2006;46:823-33.

76. Schmitz RJ, Tamada Y, Doyle MR, Zhang X, Amasino RM. Histone H2B Deubiquitination is required for transcriptional activation of FLOWERING LOCUS C and for proper control of flowering in Arabidopsis. Plant Physiol. 2008;149:1196-204.

77. Tsuji H, Taoka Kl, Shimamoto K. Regulation of flowering in rice: two florigen genes, a complex gene network, and natural variation. Curr Opin Plant Biol. 2011;14:45-52.

78. Hecht V, Laurie R, Vander Schoor J, Ridge S, Knowles C, Liew L, et al. The pea GIGAS gene is a FLOWERING LOCUS T homolog necessary for grafttransmissible specification of flowering but not for responsiveness to photoperiod. Plant Cell. 2011;23:147-61.

\section{Ready to submit your research? Choose BMC and benefit from:}

- fast, convenient online submission

- thorough peer review by experienced researchers in your field

- rapid publication on acceptance

- support for research data, including large and complex data types

- gold Open Access which fosters wider collaboration and increased citations

- maximum visibility for your research: over $100 \mathrm{M}$ website views per year

At BMC, research is always in progress.

Learn more biomedcentral.com/submissions 